\title{
Yangians, Integrable Quantum Systems and Dorey's Rule
}

\author{
Vyjayanthi Chari and Andrew Pressley \\ 1. Introduction \\ 2. Yangians \\ 3. Finite dimensional representations \\ 4. Dorey's rule \\ 5. Some preliminary lemmas \\ 6. The $A_{n}$ case \\ 7. The $D_{n}$ case \\ 8. The $B_{n}$ and $C_{n}$ cases \\ 9. The quantum affine case \\ 10. Appendix: Dorey's rule and affine Toda theories \\ 11. References
}

\section{Introduction}

Quantum groups arose from the quantum inverse scattering method, developed by the Leningrad school [13] to solve integrable quantum systems. They provide, in particular, a way to understand the solutions of the quantum Yang-Baxter equation (R-matrices) associated to such systems, and a general framework for producing new solutions. Of special importance are the solutions which depend on a complex ('spectral') parameter; those which are rational, or trigonometric, functions of this parameter arise from the quantum groups called Yangians, or quantum affine algebras, respectively (see [11], [12] and Chapter 12 in [8] for background information).

More recently, quantum groups have arisen in another guise in connection with $1+1$ dimensional integrable quantum field theories, namely as the algebras satisfied by certain non-local conserved currents. For example, Yangians appear as 'quantum symmetry algebras' in $G$-invariant Wess-Zumino-Witten models [1], while quantum affine algebras appear in affine Toda field theories (ATFTs) [2]. In [10], Dorey gave a remarkable Lie-theoretic description of the classical three-point couplings (or 'fusings') in certain integrable field theories, including ATFTs. It is the purpose of this paper to interpret Dorey's rule in terms of the representation theory of Yangians and quantum affine algebras.

To describe our results in more detail, recall that an ATFT is a theory of scalar fields with exponential interactions determined by the roots of a (possibly twisted) affine Lie algebra. If $\mathfrak{g}$ is a finite-dimensional complex simple Lie algebra, and $\hat{\mathfrak{g}}$ is the associated (untwisted) affine Lie algebra, the quantum affine algebra $U_{\epsilon}(\hat{\mathfrak{g}})$ 
$\hat{\mathfrak{g}}^{*}$, whose Dynkin diagram is obtained from that of $\hat{\mathfrak{g}}$ by reversing the arrows (the deformation parameter $\epsilon$ is related to the coupling constant of the theory, which should be purely imaginary for the quantum affine symmetry to exist - see Section $10)$. Note that $\hat{\mathfrak{g}}$ is self-dual if $\mathfrak{g}$ is simply-laced, but otherwise $\hat{\mathfrak{g}}^{*}$ is a twisted affine algebra $\hat{\mathfrak{k}}^{\sigma}$, where $\mathfrak{k}$ is simply-laced and $\sigma$ is a diagram automorphism of $\mathfrak{k}$.

The manifestation of this quantum affine symmetry of interest to us is the relation, conjectured by physicists, between the so-called 'fundamental representations' of $U_{\epsilon}(\hat{\mathfrak{g}})$ and the 'fusings' of the classical and quantum particles of the ATFT based on $\hat{\mathfrak{g}}^{*}$. It is well known (see [5] and [10], for example) that the masses of the particles in the theory form the components of the eigenvector with lowest eigenvalue of the Cartan matrix of $\mathfrak{g}$; in particular, there is a natural one-to-one correspondence between these particles and the nodes of the Dynkin diagram of $\mathfrak{g}$. One says that there is a fusing between the particles labelled $i, j$ and $k$ if a certain term in the lagrangian of the theory is non-vanishing (see Section 10). Choose a colouring of the nodes of the Dynkin diagram of $\mathfrak{g}$ black or white in such a way that linked nodes have different colour, and let $\gamma$ be the Coxeter element of the Weyl group of $\mathfrak{g}$ obtained by taking the product of the simp

le reflections associated to the black nodes, followed by those associated to the white nodes. Let $R_{i}$ be the $\gamma$-orbit of the simple root $\alpha_{i}$ if $i$ is black, and of $-\alpha_{i}$ if $i$ is white. Then, Dorey's rule asserts that there is a non-trivial coupling between the particles labelled $i, j$ and $k$ if and only if

$$
0 \in R_{i}+R_{j}+R_{k}
$$

A little later, it was shown in [20] that (D) also gives the fusing rule for the solitons in the classical theory.

For the theory based on a twisted affine algebra $\hat{\mathfrak{k}}^{\sigma}$, the particles are in one-toone correspondence with the orbits of $\sigma$ on the nodes of the Dynkin diagram of $\mathfrak{k}$, and a twisted version of $(\mathrm{D})$ is required to describe their fusings. One defines a 'twisted Coxeter element' $\tilde{\gamma}$ for the pair $(\mathfrak{k}, \sigma)$, with the property that the orbits of $\tilde{\gamma}$ on the set of roots of $\mathfrak{k}$ are in one-to-one correspondence with the orbits of $\sigma$ on the nodes of the Dynkin diagram of $\mathfrak{k}$. If $\mathfrak{g}$ is the (non-simply-laced) algebra such that $\hat{\mathfrak{g}}^{*} \cong \hat{\mathfrak{k}}^{\sigma}$, these orbits are naturally in one-to-one correspondence with the nodes of the Dynkin diagram of $\mathfrak{g}$. Proceeding as above, one obtains an analogue (TD) of (D), in which the indices $i, j$ and $k$ may be viewed as nodes of the Dynkin diagram of $\mathfrak{g}$, although the analogues of the $R_{i}$ are sets of roots of $\mathfrak{k}$. Then the classical fusings of the ATFT based on $\hat{\mathfrak{g}}^{*}$, whe

re $\mathfrak{g}$ is non-simply-laced, are given by (TD).

The situation in the quantum theory turns out to be slightly different. This time, the fusings of the ATFT based on $\hat{\mathfrak{g}}^{*}$ are given by (D) if $\mathfrak{g}$ is simply-laced, but by (D) $\cap(\mathrm{TD})$ otherwise (this can be verified case-by-case using the results in [9], at least when $\mathfrak{g}$ is not of type $E$ or $F$ ).

Even without this physical motivation, (D) strongly suggests a connection to representation theory because of its similarity to the condition occurring in the Parthasarathy-Ranga Rao-Varadarajan (PRV) conjecture [21]. This conjecture, proved by Kumar [16] and Mathieu [18], asserts that, if $\mu_{1}, \mu_{2}$ and $\mu_{3}$ are dominant weights of $\mathfrak{g}, W \mu_{1}$ the Weyl group orbit of $\mu_{1}$, and $W\left(\mu_{1}\right)$ the irreducible $\mathfrak{g}$-module with highest weight $\mu_{1}$, etc., then 
implies

$$
\operatorname{Hom}_{\mathfrak{g}}\left(W\left(\mu_{1}\right) \otimes W\left(\mu_{2}\right) \otimes W\left(\mu_{3}\right), \mathbb{C}\right) \neq 0
$$

( $\mathbb{C}$ denotes the one-dimensional trivial $\mathfrak{g}$-module). Now, as Braden [4] pointed out, (D) is equivalent to

$$
0 \in \Gamma \lambda_{i}+\Gamma \lambda_{j}+\Gamma \lambda_{k}
$$

where $\Gamma$ is the cyclic subgroup of $W$ generated by $\gamma$, so (D) is obtained from (PRV) by replacing $W$ by $\Gamma$ (and restricting to fundamental weights).

The fundamental representations of $U_{\epsilon}(\hat{\mathfrak{g}})$ to which (D) is related can be characterised as the finite-dimensional irreducible representations of $U_{\epsilon}(\hat{\mathfrak{g}})$ which contain a fundamental representation $W_{\epsilon}\left(\lambda_{i}\right)$ of $U_{\epsilon}(\mathfrak{g})$, and are such that all other irreducible $U_{\epsilon}(\mathfrak{g})$-subrepresentations have highest weight strictly less than $\lambda_{i}$ (see [7] and [8]). There is, in fact, a family of such representations $V\left(\lambda_{i}, a\right)$ of $U_{\epsilon}(\hat{\mathfrak{g}})$, depending on a parameter $a \in \mathbb{C}^{\times}$. The representations $V\left(\lambda_{i}, a\right)$ and $V\left(\lambda_{i}, b\right)$ are related by twisting by an automorphism of $U_{\epsilon}(\hat{\mathfrak{g}})$ which fixes $U_{\epsilon}(\mathfrak{g})$ and corresponds, at the classical level, to the automorphism of the loop algebra $\mathfrak{g}\left[t, t^{-1}\right]$ which sends $t$ to $a t / b$ (the central extension by which $\hat{\mathfrak{g}}$ is obtained from the loop algebra plays no role here, since it acts trivially on all the representations of interest). Now recall that, whether or not $\mathfrak{g}$ is

simply-laced, the particles of the ATFT based on $\hat{\mathfrak{g}}^{*}$ are in one-to-one correspondence with the nodes of the Dynkin diagram of $\mathfrak{g}$. If $i, j$ and $k$ are three such nodes, we would therefore expect a fusing between the quantum particles labelled $i, j$ and $k$ if and only if

$$
\operatorname{Hom}_{U_{\epsilon}(\hat{\mathfrak{g}})}\left(V\left(\lambda_{i}, a\right) \otimes V\left(\lambda_{j}, b\right) \otimes V\left(\lambda_{k}, c\right), \mathbb{C}\right) \neq 0,
$$

for some $a, b, c \in \mathbb{C}^{\times}$. Thus, ( $\otimes$ ) should hold if and only if $i, j$ and $k$ satisfy (D) when $\mathfrak{g}$ is simply-laced, or (D) $\cap(\mathrm{TD})$ otherwise. This conjecture was first made explicit by MacKay [17].

In this paper, we prove this conjecture when $\mathfrak{g}$ is not of exceptional type. We also prove an analogous result for Yangians (it was actually in the context of Yangians that MacKay originally made his conjecture). In fact, in the body of the paper, we concentrate on the Yangian case, and describe at the end how to translate the main results from the context of Yangians to that of quantum affine algebras. As MacKay has emphasized [17], the truth of the conjecture indicates that there is some beautiful structure in the representation theory of $U_{\epsilon}(\hat{\mathfrak{g}})$ which is not evident at our present state of knowledge. It also suggests that it would be interesting to study the representation theory of twisted quantum affine algebras, but this does not seem to have been attempted yet.

One approach to the conjecture is through R-matrices. There is a canonical $\operatorname{map} R(a, b) \in \operatorname{End}\left(V\left(\lambda_{i}, a\right) \otimes V\left(\lambda_{j}, b\right)\right)$ which is a rational function of the spectral parameter $a / b$, and is such that $\tau R(a, b)$ commutes with the action of $U_{\epsilon}(\hat{\mathfrak{g}})(\tau$ denotes the flip of the two factors in the tensor product). In some cases, explicit formulas for $R(a, b)$ (or rather its Yangian analogue) were given in [7] (and earlier in [19], but without proper mathematical justification). There is a finite set of values of $a / b$ for which $R(a, b)$ is well defined, but not invertible, and then its kernel is a subrepresentation of $V\left(\lambda_{i}, a\right) \otimes V\left(\lambda_{j}, b\right)$. If one can choose $a / b$ so that this subrepresentation is fundamental, one deduces that $(\otimes)$ holds for some $k, c$. To use 
to compute the R-matrix associated to every pair of fundamental representations; in addition, one would have to prove that every fundamental subrepresentation of $V\left(\lambda_{i}, a\right) \otimes V\left(\lambda_{j}, b\right)$ arises from

the R-matrix as above. Because of these difficulties, we employ a different and simpler method, which makes no use of R-matrices, and which establishes the reverse implication at the same time.

Acknowledgements We thank Harry Braden and Niall MacKay for drawing our attention to this problem, and Gustav Delius, Mike Freeman and Patrick Dorey for several illuminating discussions concerning ATFTs, and T. A. Springer for help with Coxeter elements, especially in the twisted case.

\section{Yangians}

Let $\mathfrak{g}$ be a finite-dimensional complex semisimple Lie algebra with Cartan subalgebra $\mathfrak{h}$ and Cartan matrix $A=\left(a_{i j}\right)_{i, j \in I}$. Fix coprime positive integers $\left(d_{i}\right)_{i \in I}$ such that the matrix $\left(d_{i} a_{i j}\right)$ is symmetric. Let $R$ be the set of roots, $R^{+}$a set of positive roots, and $R^{-}=-R^{+}$. The roots can be regarded as functions $I \rightarrow \mathbb{Z}$; in particular, the simple roots $\alpha_{i} \in R^{+}$are given by

$$
\alpha_{i}(j)=a_{j i}, \quad(i, j \in I)
$$

Let $Q=\oplus_{i \in I} \mathbb{Z} \cdot \alpha_{i} \subset \mathfrak{h}^{*}$ be the root lattice, and set $Q^{+}=\sum_{i \in I} \mathbb{N} \cdot \alpha_{i}$.

A weight is an arbitrary function $\lambda: I \rightarrow \mathbb{Z}$; denote the set of weights by $P$, and let

$$
P^{+}=\{\lambda \in P: \lambda(i) \geq 0 \text { for all } i \in I\}
$$

be the set of dominant weights. Define a partial order $\geq$ on $P$ by

$$
\lambda \geq \mu \text { if and only if } \lambda-\mu \in Q^{+} .
$$

Let $\theta$ be the unique highest root with respect to $\geq$.

Let $($, ) be the non-degenerate invariant symmetric bilinear form on $\mathfrak{g}$ such that the induced form on $\mathfrak{h}^{*}$ is given by

$$
\left(\alpha_{i}, \alpha_{j}\right)=d_{i} a_{i j}
$$

If $\beta \in R$, set $d_{\beta}=\frac{1}{2}(\beta, \beta)$. Let $W$ be the Weyl group of $\mathfrak{g}$, let $\left\{s_{i}\right\}_{i \in I}$ be the simple reflections which generate it, and let $w_{0}$ be the longest element of $W$. The dual Coxeter number $\breve{h}$ of $\mathfrak{g}$ is

$$
\check{h}=1+2 \frac{(\rho, \theta)}{(\theta, \theta)},
$$

where $\rho$ is half the sum of the positive roots of $\mathfrak{g}$.

Fix a basis $\left\{H_{i}\right\}_{i \in I} \cup\left\{X_{\alpha}^{ \pm}\right\}_{\alpha \in R^{+}}$of $\mathfrak{g}$ such that, for all $i \in I, \alpha, \beta \in R^{+}$,

$$
\left[H_{i}, X_{\alpha}^{ \pm}\right]= \pm \alpha(i) X_{\alpha}^{ \pm}, \quad\left[X_{\alpha}^{+}, X_{\beta}^{-}\right]=\delta_{\alpha, \beta} H_{\alpha}
$$


where $H_{\alpha}=\sum_{i} n_{i} H_{i}$ if $\alpha=\sum_{i} n_{i} \alpha_{i}$. Let $X_{i}^{ \pm}=X_{\alpha_{i}}^{ \pm}$.

If $\left\{I_{p}\right\}$ is an orthonormal basis of $\mathfrak{g}$ with respect to $($,$) , let$

$$
\Omega=\sum_{p} I_{p}^{2}
$$

be the Casimir element of the universal enveloping algebra $U(\mathfrak{g})$. We also denote by $\Omega$ the element

$$
\Omega=\sum_{p} I_{p} \otimes I_{p} \in \mathfrak{g} \otimes \mathfrak{g}
$$

Let $\kappa$ be $1 / 4$ of the value of $\Omega$ acting in the adjoint representation of $\mathfrak{g}$ (the value of $\kappa$ is given in Section 3).

Definition 2.1. ([11]) The Yangian $Y(\mathfrak{g})$ is the algebra over $\mathbb{C}$ generated by elements $x, J(x)$, for $x \in \mathfrak{g}$, with the following defining relations:

$$
\begin{gathered}
{[x, y] \quad(\text { in } Y(\mathfrak{g}))=[x, y] \quad(\text { in } \mathfrak{g}),} \\
J(a x+b y)=a J(x)+b J(y), \\
{[x, J(y)]=J([x, y]),} \\
{[J(x), J([y, z])]+[J(z), J([x, y])]+[J(y), J([z, x])]=} \\
\sum_{p, q, r}\left(\left[x, I_{p}\right],\left[\left[y, I_{q}\right],\left[z, I_{r}\right]\right]\right)\left\{I_{p}, I_{q}, I_{r}\right\}, \\
{[[J(x), J(y)],[z, J(w)]]+[[J(z), J(w)],[x, J(y)]]=} \\
\sum_{p, q, r}\left(\left[x, I_{p}\right],\left[\left[y, I_{q}\right],\left[[z, w], I_{r}\right]\right]\right)\left\{I_{p}, I_{q}, J\left(I_{r}\right)\right\},
\end{gathered}
$$

for all $x, y, z \in \mathfrak{g}, a, b \in \mathbb{C}$. Here, for any elements $z_{1}, z_{2}, z_{3} \in Y(\mathfrak{g})$, we set

$$
\left\{z_{1}, z_{2}, z_{3}\right\}=\frac{1}{24} \sum_{\pi} z_{\pi(1)} z_{\pi(2)} z_{\pi(3)}
$$

the sum being over all permutations $\pi$ of $\{1,2,3\}$.

The Yangian $Y(\mathfrak{g})$ has a Hopf algebra structure with counit $\epsilon$, comultiplication $\Delta$ and antipode $S$ given by

$$
\begin{aligned}
\Delta(x) & =x \otimes 1+1 \otimes x, \\
\Delta(J(x)) & =J(x) \otimes 1+1 \otimes J(x)+\frac{1}{2}[x \otimes 1, \Omega], \\
S(x) & =-x, \quad S(J(x))=-J(x)+\kappa x, \\
\epsilon(x) & =\epsilon(J(x))=0 .
\end{aligned}
$$

We shall also need the following presentation of $Y(\mathfrak{g})$, given in [12]:

Theorem 2.2. The Yangian $Y(\mathfrak{g})$ is isomorphic to the associative algebra with generators $X_{i, r}^{ \pm}, H_{i, r}, i \in I, r \in \mathbb{N}$, and the following defining relations: 


$$
\begin{aligned}
{\left[H_{i, 0}, X_{j, s}^{ \pm}\right] } & = \pm d_{i} a_{i j} X_{j, s}^{ \pm}, \\
{\left[H_{i, r+1}, X_{j, s}^{ \pm}\right]-\left[H_{i, r}, X_{j, s+1}^{ \pm}\right] } & = \pm \frac{1}{2} d_{i} a_{i j}\left(H_{i, r} X_{j, s}^{ \pm}+X_{j, s}^{ \pm} H_{i, r}\right), \\
{\left[X_{i, r}^{+}, X_{j, s}^{-}\right] } & =\delta_{i, j} H_{i, r+s}, \\
{\left[X_{i, r+1}^{ \pm}, X_{j, s}^{ \pm}\right]-\left[X_{i, r}^{ \pm}, X_{j, s+1}^{ \pm}\right] } & = \pm \frac{1}{2} d_{i} a_{i j}\left(X_{i, r}^{ \pm} X_{j, s}^{ \pm}+X_{j, s}^{ \pm} X_{i, r}^{ \pm}\right), \\
\sum_{\pi}\left[X_{i, r_{\pi(1)}}^{ \pm},\left[X_{i, r_{\pi(2)}}^{ \pm}, \ldots,\left[X_{i, r_{\pi(m)}}^{ \pm}, X_{j, s}^{ \pm}\right] \cdots\right]\right] &
\end{aligned}
$$

for all sequences of non-negative integers $r_{1}, \ldots, r_{m}$, where $m=1-a_{i j}$ and the sum is over all permutations $\pi$ of $\{1, \ldots, m\}$.

The isomorphism $f$ between the two realizations of $Y(\mathfrak{g})$ is given by

$$
\begin{aligned}
f\left(H_{i}\right) & =d_{i}^{-1} H_{i, 0}, \quad f\left(J\left(H_{i}\right)\right)=d_{i}^{-1} H_{i, 1}+f\left(v_{i}\right), \\
f\left(X_{i}^{ \pm}\right) & =X_{i, 0}^{ \pm}, \quad f\left(J\left(X_{i}^{ \pm}\right)\right)=X_{i, 1}^{ \pm}+f\left(w_{i}^{ \pm}\right),
\end{aligned}
$$

where

$$
\begin{aligned}
v_{i} & =\frac{1}{4} \sum_{\beta \in \Delta^{+}} \frac{d_{\beta}}{d_{i}}\left(\beta, \alpha_{i}\right)\left(X_{\beta}^{+} X_{\beta}^{-}+X_{\beta}^{-} X_{\beta}^{+}\right)-\frac{d_{i}}{2} H_{i}^{2} \\
w_{i}^{ \pm} & = \pm \frac{1}{4} \sum_{\beta \in \Delta^{+}} d_{\beta}\left(\left[X_{i}^{ \pm}, X_{\beta}^{ \pm}\right] X_{\beta}^{\mp}+X_{\beta}^{\mp}\left[X_{i}^{ \pm}, X_{\beta}^{ \pm}\right]\right)-\frac{1}{4} d_{i}\left(X_{i}^{ \pm} H_{i}+H_{i} X_{i}^{ \pm}\right) .
\end{aligned}
$$

Remarks. 1. The presentation 2.1 of $Y(\mathfrak{g})$ shows that there is a canonical map $\mathfrak{g} \rightarrow Y(\mathfrak{g})$ (it is known that this map is injective). Thus, any $Y(\mathfrak{g})$-module may be regarded as a $\mathfrak{g}$-module.

2. If $\pi$ is a permutation of $I$ such that

$$
H_{i} \mapsto H_{\pi(i)}, \quad X_{i}^{ \pm} \mapsto X_{\pi(i)}^{ \pm}
$$

defines a Lie algebra automorphism of $\mathfrak{g}$, the assignment

$$
H_{i, k} \mapsto H_{\pi(i), k}, \quad X_{i, k}^{ \pm} \mapsto X_{\pi(i), k}^{ \pm}
$$

defines a Hopf algebra automorphism of $Y(\mathfrak{g})$. We denote both of these automorphisms simply by $\pi$. 
Proposition 2.3. There is a one-parameter group $\left\{\tau_{a}\right\}_{a \in \mathbb{C}}$ of Hopf algebra automorphisms of $Y(\mathfrak{g})$ given in terms of the presentation 2.1 by

$$
\tau_{a}(x)=x, \quad \tau_{a}(J(x))=J(x)+a x,
$$

for $x \in \mathfrak{g}$, and in terms of the presentation 2.2 by

$$
\tau_{a}\left(H_{i, k}\right)=\sum_{r=0}^{k}\left(\begin{array}{l}
k \\
r
\end{array}\right) a^{k-r} H_{i, r}, \quad \tau_{a}\left(X_{i, k}^{ \pm}\right)=\sum_{r=0}^{k}\left(\begin{array}{l}
k \\
r
\end{array}\right) a^{k-r} X_{i, r}^{ \pm} .
$$

This is Proposition 2.6 in [7].

The second automorphism is an extension of the Cartan involution

$$
\varphi_{0}\left(H_{i}\right)=-H_{i}, \quad \varphi_{0}\left(X_{i}^{ \pm}\right)=X_{i}^{\mp}
$$

of $\mathfrak{g}$ to $Y(\mathfrak{g})$.

Proposition 2.4. There exists a unique algebra automorphism $\varphi$ of $Y(\mathfrak{g})$ such that

$$
\varphi\left(H_{i, k}\right)=(-1)^{k+1} H_{i, k}, \quad \varphi\left(X_{i, k}^{ \pm}\right)=(-1)^{k} X_{i, k}^{\mp},
$$

for all $i \in I, k \in \mathbb{N}$. Moreover, $\varphi$ is a coalgebra anti-automorphism of $Y(\mathfrak{g})$.

Proof. It is easy to check that applying $\varphi$ to one of the defining relations in 2.2 gives another of the defining relations. Hence, the assignment in the statement of the proposition extends uniquely to an algebra homomorphism $Y(\mathfrak{g}) \rightarrow Y(\mathfrak{g})$, and it is obvious that $\varphi$ is an involution.

Using the isomorphism $f$ in 2.2 , it is clear that $\left.\varphi\right|_{\mathfrak{g}}=\varphi_{0}$ and that

$$
\varphi\left(J\left(H_{i}\right)\right)=J\left(H_{i}\right) .
$$

Hence,

$$
\varphi\left(J\left(X_{i}^{ \pm}\right)\right)=\mp \frac{1}{2} \varphi\left(\left[X_{i}^{ \pm}, J\left(H_{i}\right)\right]\right)=\mp \frac{1}{2}\left[X_{i}^{\mp}, J\left(H_{i}\right)\right]=-J\left(X_{i}^{\mp}\right) .
$$

To prove that

$$
(\varphi \otimes \varphi) \circ \Delta=\Delta^{\mathrm{op}} \circ \varphi,
$$

where $\Delta^{\text {op }}$ denotes the opposite comultiplication of $Y(\mathfrak{g})$, it suffices to show that both sides agree when applied to a set of generators of $Y(\mathfrak{g})$, such as $\left\{H_{i}, X_{i}^{ \pm}, J\left(H_{i}\right), J\left(X_{i}^{ \pm}\right)\right\}_{i \in I}$. This is now straightforward, making use of the formula for $\Delta$ in 2.1 and the observation that $\left(\varphi_{0} \otimes \varphi_{0}\right)(\Omega)=\Omega$.

We shall also need the following weak version of the Poincaré-Birkhoff-Witt theorem for $Y(\mathfrak{g})$.

Proposition 2.5. Let $Y^{+}, Y^{-}$and $Y^{0}$ be the subalgebras of $Y(\mathfrak{g})$ generated by the $X_{i, k}^{+}$, the $X_{i, k}^{-}$and the $H_{i, k}$, respectively $(i \in I, k \in \mathbb{N})$. Then,

$$
Y(\mathfrak{g})=Y^{-} \cdot Y^{0} \cdot Y^{+}
$$




\section{Finite-dimensional representations}

If $W$ is a $\mathfrak{g}$-module and $\lambda \in P$, the weight space

$$
W_{\lambda}=\left\{w \in W \mid H_{i} . w=\lambda(i) w \text { for all } i \in I\right\}
$$

If $W_{\lambda} \neq 0, \lambda$ is called a weight of $W$, and the set of such weights is denoted by $P(W)$.

A non-zero vector $w \in W$ is called a $\mathfrak{g}$-highest weight vector if $w \in W_{\lambda}$ for some $\lambda \in P(W)$ and $X_{i}^{+} . w=0$ for all $i \in I$. Let $W^{+}$be the set of $\mathfrak{g}$-highest weight vectors of $W$, and set $W_{\lambda}^{+}=W^{+} \cap W_{\lambda}$. If $W=U(\mathfrak{g}) . w$, then $W$ is called a highest weight $\mathfrak{g}$-module with highest weight $\lambda$. Lowest weight vectors and $\mathfrak{g}$-modules are defined similarly. For any $\lambda \in P^{+}$denote by $W(\lambda)$ the unique irreducible highest weight $\mathfrak{g}$-module with highest weight $\lambda$. If $W$ is any finite-dimensional $\mathfrak{g}$-module, we have

$$
W \cong \bigoplus_{\lambda \in P^{+}} W(\lambda)^{\oplus m_{\lambda}(W)}
$$

where the multiplicities $m_{\lambda}(W)$ are given by

$$
m_{\lambda}(W)=\operatorname{dim}\left(W^{+} \cap W_{\lambda}\right)
$$

We recall that the Casimir operator $\Omega \in U(\mathfrak{g})$ acts on $W(\lambda)$ by the scalar $(\lambda+2 \rho, \lambda)$. In particular, $\kappa=\frac{1}{2} d_{\theta} \breve{h}$.

Let $W^{*}$ be the dual $\mathfrak{g}$-module of $W$, and let $W_{0}^{\varphi}$ be the $\mathfrak{g}$-module obtained by twisting $W$ with the Cartan involution $\varphi_{0}$ of $\mathfrak{g}$. For $\lambda \in P$, let $\bar{\lambda}=-w_{0}(\lambda)$. It is well known that

$$
m_{\lambda}(W)=m_{\bar{\lambda}}\left(W^{*}\right)=m_{\bar{\lambda}}\left(W^{\varphi_{0}}\right)
$$

Suppose now that $V$ is a $Y(\mathfrak{g})$-module. Set

$$
V^{++}=\left\{v \in V^{+} \mid X_{i, k}^{+} \cdot v=0 \text { for all } i \in I, k \in \mathbb{N}\right\}
$$

and for any $\lambda \in P^{+}$, set $V_{\lambda}^{++}=V^{++} \cap V_{\lambda}$. Note that, by $2.2, V^{++}$is preserved by the action of $Y^{0}$, and so, if $V^{++} \neq 0$, it contains a non-zero $Y^{0}$-eigenvector $v$ (say), so that

$$
H_{i, k} \cdot v=d_{i, k} v
$$

for some $d_{i, k} \in \mathbb{C}$. Such a vector $v$ is called a $Y(\mathfrak{g})$-highest weight vector, $V$ is called $Y(\mathfrak{g})$-highest weight if $V=Y(\mathfrak{g}) \cdot v$ for some $Y(\mathfrak{g})$-highest weight vector $v \in V$, and the collection of scalars $\mathbf{d}=\left\{d_{i, k}\right\}_{i \in I, k \in \mathbb{N}}$ is called its highest weight. It is not difficult to show that, for every $\mathbf{d}=\left(d_{i, k}\right)_{i \in I, k \in \mathbb{N}}$, there is an irreducible $Y(\mathfrak{g})$ module $V(\mathbf{d})$, unique up to isomorphism, such that $V(\mathbf{d})$ has highest weight $\mathbf{d}$. Lowest weight vectors and modules for $Y(\mathfrak{g})$ are defined similarly.

The following theorem of Drinfel'd [12] classifies the finite-dimensional irreducible 
Theorem 3.1. (i) Every finite-dimensional irreducible $Y(\mathfrak{g})$-module is both highest weight and lowest weight.

(ii) If $\mathbf{d}=\left(d_{i, k}\right)_{i \in I, k \in \mathbb{N}}$, the $Y(\mathfrak{g})$-module $V(\mathbf{d})$ is finite-dimensional if and only if there exist monic polynomials $P_{i} \in \mathbb{C}[u]$ such that

$$
\frac{P_{i}\left(u+d_{i}\right)}{P_{i}(u)}=1+\sum_{k=0}^{\infty} d_{i, k} u^{-k-1},
$$

in the sense that the right-hand side is the Laurent expansion of the left-hand side about $u=\infty$.

If $V$ is a finite-dimensional irreducible $Y(\mathfrak{g})$-module, we call the associated $I$ tuple of polynomials $\left(P_{i}\right)_{i \in I}$ the Drinfel'd polynomials of $V$.

In general, if $V$ is any finite-dimensional $Y(\mathfrak{g})$-module and $v \in V$ is a $Y(\mathfrak{g})$ highest weight vector, with

$$
H_{i, k} v=d_{i, k}^{v} v
$$

for some $d_{i, k}^{v} \in \mathbb{C}$, it follows from 3.1 that there exist monic polynomials $P_{i}^{v}$ such that

$$
\frac{P_{i}^{v}\left(u+d_{i}\right)}{P_{i}^{v}(u)}=1+\sum_{k=0}^{\infty} d_{i, k}^{v} u^{-k-1}
$$

Proposition 3.2. Let $V_{1}, V_{2}$ be finite-dimensional $Y(\mathfrak{g})$-modules, and let $v_{1} \in V_{1}$, $v_{2} \in V_{2}$ be $Y(\mathfrak{g})$-highest weight vectors. Then,

$$
P_{i}^{v_{1} \otimes v_{2}}=P_{i}^{v_{1}} P_{i}^{v_{2}}
$$

This is Proposition 2.15 in [7].

The $Y(\mathfrak{g})$-modules of interest in this paper are defined as follows.

Definition 3.3. If $i \in I, a \in \mathbb{C}$, then $V_{a}\left(\lambda_{i}\right)$ is the finite-dimensional irreducible $Y(\mathfrak{g})$-module with Drinfel'd polynomials

$$
P_{j}(u)= \begin{cases}u-a & \text { if } j=i, \\ 1 & \text { if } j \neq i .\end{cases}
$$

We call $V_{a}\left(\lambda_{i}\right)$ a fundamental $Y(\mathfrak{g})$-module.

Given a finite-dimensional $Y(\mathfrak{g})$-module $V$, we can define the following associated $Y(\mathfrak{g})$-modules:

(i) $V(a)$ : this is obtained pulling back $V$ through $\tau_{a}$;

(ii) $V^{\varphi}$ : this is obtained pulling back $V$ through $\varphi$;

(iii) the left dual ${ }^{t} V$ and right dual $V^{t}$ : these are given by the following actions of $Y(\mathfrak{g})$ on the vector space dual of $V$ :

$$
\begin{aligned}
& (y \cdot f)(v)=f(S(y) \cdot v), \quad y \in Y(\mathfrak{g}), f \in{ }^{t} V, v \in V, \\
& (y \cdot f)(v)=f\left(S^{-1}(y)\right) . v, \quad y \in Y(\mathfrak{g}), f \in V^{t}, v \in V .
\end{aligned}
$$


Proposition 3.4.. Let $U, V$ and $W$ be finite-dimensional $Y(\mathfrak{g})$-modules, and let $a \in \mathbb{C}$. Then,

(i) $(U \otimes V)^{\varphi} \cong V^{\varphi} \otimes U^{\varphi}$;

(ii) $V(a)^{\varphi} \cong V^{\varphi}(-a)$;

(iii) $\operatorname{Hom}_{Y(\mathfrak{g})}(U, V \otimes W) \cong \operatorname{Hom}_{Y(\mathfrak{g})}\left({ }^{t} V \otimes U, W\right)$;

(iv) $\operatorname{Hom}_{Y(\mathfrak{g})}(U, W \otimes V) \cong \operatorname{Hom}_{Y(\mathfrak{g})}\left(U \otimes V^{t}, W\right)$;

(v) ${ }^{t t} V \cong V(-2 \kappa), V^{t t} \cong V(2 \kappa),{ }^{t}\left(V^{t}\right) \cong\left({ }^{t} V\right)^{t} \cong V$;

(vi) $(V \otimes W)^{t} \cong\left(W^{t} \otimes V^{t}\right),{ }^{t}(V \otimes W) \cong{ }^{t} W \otimes{ }^{t} V$;

(vii) $(V(a))^{t} \cong V^{t}(a),{ }^{t}(V(a)) \cong\left({ }^{t} V\right)(a)$.

Proof. Part (i) follows from the fact that $\varphi$ is a coalgebra anti-automorphism of $Y(\mathfrak{g})$, and part (ii) from the identity

$$
\varphi \cdot \tau_{a}=\tau_{-a} \cdot \varphi
$$

which is proved by checking that the two sides agree when applied to any of the generators $H_{i, k}, X_{i, k}^{ \pm}$. Parts (iii)-(vii) are straightforward.

The following result describes the Drinfel'd polynomials of the modules defined above. If $i \in I$, define $\bar{i} \in I$ by $\lambda_{\bar{i}}=\overline{\lambda_{i}}$.

Proposition 3.5. Let $V$ be a finite-dimensional irreducible $Y(\mathfrak{g})$-module with Drinfel'd polynomials $P_{i}(i \in I)$, and let $a \in \mathbb{C}$. Then:

(i) The Drinfel'd polynomials $P_{i}^{a}$ of $V(a)$ are given by

$$
P_{i}^{a}(u)=P_{i}(u-a) .
$$

(ii) The Drinfel'd polynomials ${ }^{t} P_{i}$ and $P_{i}^{t}$ of ${ }^{t} V$ and $V^{t}$, respectively, are given by

$$
{ }^{t} P_{i}(u)=P_{\bar{i}}(u+\kappa), \quad P_{i}^{t}(u)=P_{\bar{i}}(u-\kappa) .
$$

(iii) The Drinfel'd polynomials $P_{i}^{\varphi}$ of $V^{\varphi}$ are given by

$$
P_{i}^{\varphi}(u)=(-1)^{\operatorname{deg}\left(P_{i}\right)} P_{\bar{i}}\left(\kappa+d_{i}-u\right) .
$$

Proof. Parts (i) and (ii) were proved in [7]. We now prove part (iii). Let $0 \neq v \in V$ be a $Y(\mathfrak{g})$-lowest weight vector, and let

$$
H_{i, k} \cdot v=\overline{d_{i, k}} v, \quad\left(\overline{d_{i, k}} \in \mathbb{C}\right) .
$$

Then, $v$ is a $Y(\mathfrak{g})$-highest weight vector in $V^{\varphi}$ and, in $V^{\varphi}$, we have, by 2.4,

$$
H_{i, k} \cdot v=(-1)^{k+1} \overline{d_{i, k}} v \text {. }
$$

Hence, the Drinfel'd polynomials $P_{i}^{\varphi}$ of $V^{\varphi}$ satisfy

$$
\frac{P_{i}^{\varphi}\left(u+d_{i}\right)}{P_{i}^{\varphi}(u)}=1+\sum_{k=0}^{\infty}(-1)^{k+1} \overline{d_{i, k}} u^{-k-1} .
$$

On the other hand, by Propositions 3.1 and 3.2 in [7],

$$
\frac{P_{\bar{i}}(u-\kappa)}{P_{\bar{i}}\left(u+d_{i}-\kappa\right)}=1+\sum_{k=0}^{\infty} \overline{d_{i, k}} u^{-k-1} .
$$

The result follows on comparing (19) and (20). 
Corollary 3.6. Let $i \in I, a \in \mathbb{C}$. Then:

(i) ${ }^{t}\left(V_{a}\left(\lambda_{i}\right)\right) \cong V_{a-\kappa}\left(\lambda_{\bar{i}}\right), \quad\left(V_{a}\left(\lambda_{i}\right)\right)^{t} \cong V_{a+\kappa}\left(\lambda_{\bar{i}}\right)$;

(ii) $\left(V_{a}\left(\lambda_{i}\right)\right)^{\varphi} \cong V_{\kappa+d_{i}-a}\left(\lambda_{\bar{i}}\right)$.

We shall also need the following result.

Proposition 3.7. Let $V$ be a finite-dimensional highest weight $Y(\mathfrak{g})$-module. Then, $V^{\varphi}$ is also a highest weight $Y(\mathfrak{g})$-module.

Proof. Let $0 \neq v \in V_{\lambda}\left(\lambda \in P^{+}\right)$be a $Y(\mathfrak{g})$-highest weight vector. By $2.5, m_{\lambda}(V)=$ 1 and $m_{\mu}(V)=0$ unless $\mu \leq \lambda$. Let $W$ be the $\mathfrak{g}$-submodule of $V$ of type $W(\lambda)$; then $v \in W$. Let $v^{-}$be a lowest weight vector (for $\mathfrak{g}$ ) in $W$. Then, $v^{-}$is a $Y(\mathfrak{g})$-highest weight vector in $V^{\varphi}$ and

$$
Y(\mathfrak{g}) \cdot v^{-} \supset U(\mathfrak{g}) \cdot v^{-}=W
$$

so $v \in Y(\mathfrak{g}) \cdot v^{-}$, and hence

$$
V^{\varphi}=Y(\mathfrak{g}) \cdot v \subset Y(\mathfrak{g}) \cdot v^{-} .
$$

We conclude this section with the following results.

Proposition 3.8. Let $V$ be a finite-dimensional $Y(\mathfrak{g})$-module. Then, $V$ is irreducible if and only if $V$ and ${ }^{t} V$ (resp. $V$ and $V^{t}$ ) are both highest weight $Y(\mathfrak{g})$ modules.

Proof. The 'only if' part follows from 3.1 (i). For the converse, suppose that $V$ and ${ }^{t} V$ are highest weight (the other case is identical). Let $v \in V_{\lambda}\left(\lambda \in P^{+}\right)$be a $Y(\mathfrak{g})$-highest weight vector. Let $0 \neq W$ be an irreducible $Y(\mathfrak{g})$-submodule of $V$, and let $\mu$ (say) be the highest weight of $W$ as a $\mathfrak{g}$-module; thus, $\mu \leq \lambda$. Then, ${ }^{t} W$ is a quotient of ${ }^{t} V$, and these $\mathfrak{g}$-modules have maximal weights $\bar{\mu}$ and $\bar{\lambda}$, respectively (cf. the proof of 3.7). Since ${ }^{t} V$ is a $Y(\mathfrak{g})$-highest weight module, its highest weight vector must map to a non-zero element of ${ }^{t} W$. Hence, $\bar{\lambda} \leq \bar{\mu}$, so $\lambda \leq \mu$. Thus, $\lambda=\mu$ and $W=V$.

Along similar lines, we have the following result whose simple proof we omit.

Proposition 3.9. Let $V$ be a finite-dimensional $Y(\mathfrak{g})$-module, and assume that, as a $\mathfrak{g}$-module, $V$ has a unique maximal weight $\lambda \in P^{+}$. Then, $Y(\mathfrak{g})$.v is a proper submodule of $V$ if and only if $V^{t}$ (resp. $\left.{ }^{t} V\right)$ contains a $Y(\mathfrak{g})$-highest weight vector of weight strictly less than $\bar{\lambda}$.

\section{Dorey's rule}

Let $s_{1}, s_{2}, \ldots, s_{n}$ be the simple reflections in the Weyl group $W$ of $\mathfrak{g}$ (in some order), and let $\gamma=s_{1} s_{2} \ldots s_{n}$ be the associated Coxeter element of $W$. Define positive roots

$$
\phi_{i}=s_{n} s_{n-1} \ldots s_{i+1}\left(\alpha_{i}\right)
$$

and let $R_{i}$ be the $\gamma$-orbit of $\phi_{i}$. It is known that the $\phi_{i}$ are precisely the positive roots which become negative under the action of $\gamma$, and that each $R_{i}$ contains 
Definition 4.1. If $p \geq 2$, we say that indices $i_{1}, i_{2}, \ldots, i_{p} \in I$ satisfy condition $\left(\mathrm{D}_{p}\right)$ if and only if $0 \in R_{i_{1}}+R_{i_{2}}+\cdots+R_{i_{p}}$.

Note that the condition $\left(\mathrm{D}_{p}\right)$ appears to depend on a number of arbitrary choices: we had to pick a Cartan subalgebra $\mathfrak{h}$, a set of positive roots $R^{+}$, and an ordering of the set of simple reflections. However, we have

Proposition 4.2. For any $p \geq 2$, the condition $\left(\mathrm{D}_{p}\right)$ is independent of the choices made.

Proof. Let $G$ be a (connected, complex) Lie group with Lie algebra $\mathfrak{g}$. If $\overline{\mathfrak{h}}$ is another Cartan subalgebra, and $\bar{R}^{+}$a set of positive roots with respect to $\overline{\mathfrak{h}}$, there exists $g \in G$ such that $\overline{\mathfrak{h}}=\operatorname{Ad}(g)(\mathfrak{h})$ and $\bar{R}^{+}=\operatorname{Ad}(g)^{*}\left(R^{+}\right)$. Then, the $\bar{\alpha}_{i}=\operatorname{Ad}(g)^{*}\left(\alpha_{i}\right)$ are the simple roots in $\bar{R}^{+}$, and the $\bar{s}_{i}=\operatorname{Ad}(g) \circ s_{i} \circ \operatorname{Ad}\left(g^{-1}\right)$ are the corresponding simple reflections. Using the Coxeter element $\bar{\gamma}=\bar{s}_{1} \bar{s}_{2} \ldots \bar{s}_{n}$, it is easy to see that, in an obvious notation, $\bar{R}_{i}=\operatorname{Ad}(g)^{*}\left(R_{i}\right)$, and it follows immediately that

$$
0 \in R_{i_{1}}+\cdots+R_{i_{p}} \quad \text { iff } \quad 0 \in \bar{R}_{i_{1}}+\cdots+\bar{R}_{i_{p}} .
$$

Thus, we may work with a fixed Cartan subalgebra and set of positive roots, and need only consider the effect of re-ordering the set of simple reflections. It is well known (see [23], Lemma 2.3) that any such re-ordering can be achieved by a sequence of moves of the following two types:

(i) $s_{1} s_{2} \ldots s_{n-1} s_{n} \mapsto s_{n} s_{1} s_{2} \ldots s_{n-1}$;

(ii) $s_{1} \ldots s_{i-1} s_{i} s_{i+1} s_{i+2} \ldots s_{n} \mapsto s_{1} \ldots s_{i-1} s_{i+1} s_{i} s_{i+2} \ldots s_{n}$, where $s_{i} s_{i+1}=s_{i+1} s_{i}$.

Thus, it suffices to prove that, if $\bar{\gamma}$ is the Coxeter element obtained from $\gamma$ by performing one of these moves, the condition $\left(\mathrm{D}_{p}\right)$ obtained by using $\bar{\gamma}$ is equivelent to that obtained using $\gamma$. Define $\bar{\phi}_{i}$ and $\bar{R}_{i}$ in the obvious way.

For a move of type (i), it is easy to see that $\bar{\phi}_{j}=s_{n}\left(\phi_{j}\right)$ if $j \neq n$, and $\bar{\phi}_{n}=$ $s_{n} \gamma^{-1}\left(\phi_{n}\right)$. Since $\bar{\gamma}=s_{n} \gamma s_{n}$, it follows that $\bar{R}_{j}=s_{n}\left(R_{j}\right)$ for all $j$. It follows as before that the condition $\left(\mathrm{D}_{p}\right)$ is unchanged.

For type (ii), $\bar{\gamma}=\gamma$ and it is clear that $\bar{\phi}_{j}=\phi_{j}$ except possibly when $j=i$ or $i+1$. But

$$
\bar{\phi}_{i}=s_{n} \ldots s_{i+2}\left(\alpha_{i}\right)=s_{n} \ldots s_{i+2} s_{i+1}\left(\alpha_{i}\right)=\phi_{i},
$$

since $s_{i+1}\left(\alpha_{i}\right)=\alpha_{i}$, and

$$
\bar{\phi}_{i+1}=s_{n} \ldots s_{i+2} s_{i}\left(\alpha_{i+1}\right)=s_{n} \ldots s_{i+2}\left(\alpha_{i+1}\right)=\phi_{i+1},
$$

since $s_{i}\left(\alpha_{i+1}\right)=\alpha_{i+1}$. Thus, $\bar{R}_{j}=R_{j}$ for all $j$.

Despite this result, it is sometimes convenient to make a particular choice of $\gamma$, as follows (see [4], [5] and [10], for example). Choose a partition

$$
I=I_{\circ} \amalg I_{\bullet}
$$

such that

$$
a_{i j}=0 \text { if } i, j \in I_{\circ} \text { or if } i, j \in I_{\bullet} .
$$

It is clear that such a partition exists and is unique up to interchanging $I_{\circ}$ and $I_{\bullet}$. Since $s_{i}$ and $s_{j}$ commute if $i, j \in I_{\circ}$ or if $i, j \in I_{\bullet}$, the Weyl group elements

$$
\gamma_{\circ}=\prod s_{i}, \quad \gamma_{\bullet}=\prod s_{i}
$$


are well defined. Then we take $\gamma=\gamma_{\circ} \gamma_{\bullet}$. Note that $\gamma_{\circ}^{2}=\gamma_{\bullet}^{2}=1$, so that $\gamma^{-1}=\gamma_{\bullet} \gamma_{\circ}$

With this choice, it is easy to see that

$$
\phi_{i}= \begin{cases}\gamma_{\bullet} \alpha_{i} & \text { if } i \in I_{\circ} \\ \alpha_{i} & \text { if } i \in I_{\bullet}\end{cases}
$$

Note that $\gamma \phi_{i}=\gamma_{\circ} \alpha_{i}=-\alpha_{i}$ if $i \in I_{\circ}$; on the other hand, if $i \in I_{\bullet}$, it is clear that $\alpha_{i}$ occurs with coefficient -1 in the root $\gamma \phi_{i}=-\gamma_{\circ} \alpha_{i}$, so $\gamma \phi_{i} \in R^{-}$. It follows that $\alpha_{i} \in R_{i}$ if $i \in I_{\bullet}$, and $-\alpha_{i} \in R_{i}$ if $i \in I_{\circ}$.

We observe next that

$$
\phi_{i}=\lambda_{i}-\gamma^{-1} \lambda_{i}
$$

Indeed, recalling that $s_{i} \lambda_{j}=\lambda_{j}$ if $i \neq j$, and $=\lambda_{i}-\alpha_{i}$ if $i=j$, the last equation is clear when $i \in I_{\bullet}$. Similarly, if $i \in I_{\circ}$, one has

$$
\lambda_{i}-\gamma \lambda_{i}=\alpha_{i}
$$

so that

$$
\phi_{i}=-\gamma^{-1}\left(\lambda_{i}-\gamma \lambda_{i}\right)=\lambda_{i}-\gamma^{-1} \lambda_{i}
$$

Hence, $\left(D_{p}\right)$ is equivalent to the condition

$$
\gamma\left(\gamma^{r_{1}} \lambda_{i_{1}}+\gamma^{r_{2}} \lambda_{i_{2}}+\cdots+\gamma^{r_{p}} \lambda_{i_{p}}\right)=\gamma^{r_{1}} \lambda_{i_{1}}+\gamma^{r_{2}} \lambda_{i_{2}}+\cdots+\gamma^{r_{p}} \lambda_{i_{p}}
$$

for some $r_{1}, r_{2}, \ldots, r_{p} \in \mathbb{Z}$. Since one is not an eigenvalue of $\gamma$ on $\mathfrak{h}^{*}$ (see [15], Lemma 8.1, for example), this last equation is equivalent to

$$
\gamma^{r_{1}} \lambda_{i_{1}}+\gamma^{r_{2}} \lambda_{i_{2}}+\cdots+\gamma^{r_{p}} \lambda_{i_{p}}=0
$$

for some $r_{1}, r_{2}, \ldots, r_{p} \in \mathbb{Z}$.

Proposition 4.3.. If $p \geq 2$ and $i_{1}, i_{2}, \ldots, i_{p} \in I$ satisfy condition $\left(\mathrm{D}_{p}\right)$, then

$$
\operatorname{Hom}_{\mathfrak{g}}\left(W\left(\lambda_{i_{1}}\right) \otimes W\left(\lambda_{i_{2}}\right) \otimes \cdots \otimes W\left(\lambda_{i_{p}}\right), \mathbb{C}\right) \neq 0 .
$$

Proof. The condition $\left(\mathrm{D}_{p}^{\prime}\right)$ is equivalent to

$$
\lambda_{\bar{i}_{1}}=w_{0} \gamma^{r_{2}-r_{1}}\left(\lambda_{i_{2}}+\gamma^{r_{3}-r_{2}} \lambda_{i_{3}}+\cdots+\gamma^{r_{p}-r_{2}} \lambda_{i_{p}}\right) \text {. }
$$

By the PRV conjecture, this implies that

$$
m_{\lambda_{\bar{i}_{1}}}\left(W\left(\lambda_{i_{2}}\right) \otimes \cdots \otimes W\left(\lambda_{i_{p}}\right)\right) \neq 0
$$

or equivalently that

$$
\operatorname{Hom}_{\mathfrak{g}}\left(W\left(\lambda_{i_{1}}\right) \otimes W\left(\lambda_{i_{2}}\right) \otimes \cdots \otimes W\left(\lambda_{i_{p}}\right), \mathbb{C}\right) \neq 0
$$

This result (and its proof) are due to Braden [4]. A generalisation of it can also be deduced from the main results of this paper, without using the PRV conjecture (see the Remark at the end of Section 8).

Proposition 4.6 below shows that the converse of 4.3 is false if $p=3$. On the 
Proposition 4.4. $\left(D_{2}\right)$ is equivalent to $\left(C G_{2}\right)$.

Proof. Since

$$
\operatorname{Hom}_{\mathfrak{g}}\left(W\left(\lambda_{i}\right) \otimes W\left(\lambda_{j}\right), \mathbb{C}\right) \neq 0
$$

if and only if $j=\bar{i}$, it suffices to prove that, for all $i \in I$,

$$
0 \in R_{i}+R_{\bar{i}}
$$

If the Coxeter number $h$ of $\mathfrak{g}$ is even, it is well known that $w_{0}=\gamma^{h / 2}$, so

$$
\alpha_{\bar{i}}=-\gamma^{h / 2} \alpha_{i} \in-R_{i}
$$

If $h$ is odd, then $\mathfrak{g}$ is of type $A_{n}$ (with $n$ even), and the result can be checked directly by explicitly computing the orbits $R_{i}$.

In the case where $\mathfrak{g}$ is not simply-laced, we shall need a twisted version of condition $\left(\mathrm{D}_{p}\right)$. For this, we recall that the dual affine Lie algebra $\hat{\mathfrak{g}}^{*}$, whose Dynkin diagram is obtained by reversing the arrows in that of the affine Lie algebra $\hat{\mathfrak{g}}$, is the twisted affine Lie algebra associated to a diagram automorphism $\sigma$ of a simplylaced algebra $\tilde{\mathfrak{g}}$ (see [14]). Following [22], choose nodes $\tilde{i}_{1}, \tilde{i}_{2}, \ldots, \tilde{i}_{n}$ of the Dynkin diagram of $\tilde{\mathfrak{g}}$, one from each orbit of $\sigma$, and define the twisted Coxeter element $\tilde{\gamma}$ of $\tilde{\mathfrak{g}}$ by

$$
\tilde{\gamma}=\tilde{s}_{\tilde{i}_{1}} \tilde{s}_{\tilde{i}_{2}} \ldots \tilde{s}_{\tilde{i}_{n}} \sigma
$$

(here, and elsewhere in this section, $\mathrm{a}^{\sim}$ is used to denote objects associated with $\tilde{\mathfrak{g}})$. Define $\operatorname{roots} \tilde{\phi}_{\tilde{i}_{r}}$ of $\tilde{\mathfrak{g}}$ by

$$
\tilde{\phi}_{\tilde{i}_{r}}=\sigma \tilde{s}_{\tilde{i}_{n}} \tilde{s}_{\tilde{i}_{n-1}} \ldots \tilde{s}_{\tilde{i}_{r+1}}\left(\tilde{\alpha}_{\tilde{i}_{r}}\right)
$$

and let $\tilde{R}_{\tilde{i}_{r}}$ be the $\tilde{\gamma}$-orbit of $\tilde{\phi}_{\tilde{i}_{r}}$. Note that there is a natural one-to-one correspondence between the set $I$ of nodes of the Dynkin diagram of $\mathfrak{g}$ and the set of orbits of $\sigma$ on the nodes of the Dynkin diagram of $\tilde{\mathfrak{g}}$. Thus, if $p \geq 2$ and $i_{1}, i_{2}, \ldots, i_{p} \in I$, the following condition makes sense:

$$
0 \in \tilde{R}_{i_{1}}+\tilde{R}_{i_{2}}+\cdots+\tilde{R}_{i_{p}}
$$

This is the twisted analogue of condition $\left(\mathrm{D}_{p}\right)$ that we shall need. As in $4.3,\left(\mathrm{D}_{p}\right)$ is independent of the choices made in defining it. It suffices to prove independence of the choice of node from each $\sigma$-orbit, for a given choice of total ordering of these orbits. Unfortunately, we have only been able to verify this by a case-by-case check.

As in 4.4, one can check that $\left(\mathrm{TD}_{2}\right)$ is equivalent to $\left(\mathrm{CG}_{2}\right)$ (and hence also to $\left.\left(\mathrm{D}_{2}\right)\right)$.

The main purpose of this paper is to study the following conjecture, first made explicit by MacKay [17] (when $p=3$ ), but implicit in the work of several authors on affine Toda field theories (see [5], [10] and [20], for example).

Conjecture 4.5. Let $p \geq 2$ and let $i_{1}, i_{2}, \ldots, i_{p} \in I$. Then, 
for some $a_{1}, a_{2}, \ldots, a_{p} \in \mathbb{C}$, if and only if $i_{1}, i_{2}, \ldots, i_{p}$ satisfy

$$
\left\{\begin{array}{l}
\left(\mathrm{D}_{p}\right) \text { when } \mathfrak{g} \text { is simply-laced, } \\
\text { both }\left(\mathrm{D}_{p}\right) \text { and }\left(\mathrm{TD}_{p}\right) \text { when } \mathfrak{g} \text { is not simply-laced. }
\end{array}\right.
$$

It follows immediately from 3.6 and 4.4 that this conjecture is true when $p=2$. To deal with the $p=3$ case, it is useful to observe that, if $(i, j, k)$ satisfies $(23)$, so does any permutation of $(i, j, k)$ (the same is obviously true of Dorey's condition)... To see this, note first that, by 3.4 and 3.6 (i), (23) holds if and only if

$$
\operatorname{Hom}_{Y(\mathfrak{g})}\left(V_{a}\left(\lambda_{i}\right) \otimes V_{b}\left(\lambda_{j}\right), V_{c-\kappa}\left(\lambda_{\bar{k}}\right)\right) \neq 0
$$

which in turn holds if and only if

$$
\operatorname{Hom}_{Y(\mathfrak{g})}\left(V_{c-2 \kappa}\left(\lambda_{k}\right) \otimes V_{a}\left(\lambda_{i}\right) \otimes V_{b}\left(\lambda_{j}\right), \mathbb{C}\right) \neq 0
$$

Thus, (23) is preserved by cyclic permutations of $(i, j, k)$. On the other hand, by 3.6 (ii), (23) is equivalent to

$$
\operatorname{Hom}_{Y(\mathfrak{g})}\left(V_{\bar{c}}\left(\lambda_{\bar{k}}\right) \otimes V_{\bar{b}}\left(\lambda_{\bar{j}}\right) \otimes V_{\bar{a}}\left(\lambda_{\bar{i}}\right), \mathbb{C}\right) \neq 0,
$$

where $\bar{a}=\kappa+d_{i}-a$, etc. But, it is known that there exists a diagram automorphism $\pi$ of $\mathfrak{g}$ such that $\pi(i)=\bar{i}$ for all $i \in I$. Twisting by the corresponding automorphism of $Y(\mathfrak{g})$ shows that $(24)$ is equivalent to

$$
\operatorname{Hom}_{Y(\mathfrak{g})}\left(V_{\bar{c}}\left(\lambda_{k}\right) \otimes V_{\bar{b}}\left(\lambda_{j}\right) \otimes V_{\bar{a}}\left(\lambda_{i}\right), \mathbb{C}\right) \neq 0
$$

Hence, (23) is also preserved by the permutation $(i, j, k) \mapsto(k, j, i)$. Since this, together with the cyclic permutations, generates the whole symmetric group on three letters, $(23)$ is preserved by all permutations of $(i, j, k)$. It follows that, in proving 4.5, we may always assume that $i, j$ and $k$ are in some fixed order.

We conclude this section by making conditions $\left(\mathrm{D}_{3}\right)$ and $\left(\mathrm{TD}_{3}\right)$ explicit when $\mathfrak{g}$ is of type $A, B, C$ or $D$.

Proposition 4.6. Let the nodes of the Dynkin diagram of $\mathfrak{g}$ be numbered as in [3], and let $1 \leq i, j \leq k \leq n$.

(a) Let $\mathfrak{g}$ be of type $A_{n}$, Then, $i, j, k$ satisfies $\left(\mathrm{D}_{3}\right)$ if and only if one of the following conditions holds:

(i) $i+j \leq n, k=n+1-(i+j)$;

(ii) $i+j>n+1, k=2 n+2-i-j$.

(b) Let $\mathfrak{g}$ be of type $B_{n}(n \geq 3)$. Then, $i, j, k$ satisfies $\left(\mathrm{D}_{3}\right)$ if and only if one of the following conditions holds:

(i) $i+j \leq n-1, k=i+j$;

(ii) $i+j \geq n+1, k=2 n-i-j$;

(iii) $i<n, j=k=n$,

and satisfies $\left(\mathrm{TD}_{3}\right)$ if and only if one of the conditions (i), (iii) or

$(\text { ii })^{\prime} i+j \geq n, k=2 n-1-i-j$ 
(c) Let $\mathfrak{g}$ be of type $C_{n}(n \geq 2)$. Then, $i, j, k$ satisfies $\left(\mathrm{D}_{3}\right)$ if and only if one of the following conditions hold:

(i) $i+j \leq n, k=i+j$;

(ii) $i+j \geq n, k=2 n-i-j$, and satisfies $\left(\mathrm{TD}_{3}\right)$ if and only if one of the conditions (i) or

(ii) $i+j \geq n+2, k=2 n+2-i-j$

holds.

(d) Let $\mathfrak{g}$ be of type $D_{n}(n \geq 4)$. Then, $i, j, k$ satisfies $\left(\mathrm{D}_{3}\right)$ if and only if one of the following conditions holds:

(i) $i+j \leq n-2, k=i+j$;

(ii) $i+j \geq n, k=2 n-i-j-2$;

(iii) $i \leq n-2, n-i$ is even, $j=k=n-1$ or $j=k=n$;

(iv) $i \leq n-2, n-i$ is odd, $j=n-1, k=n$.

Remarks. 1. It is interesting to note that, in each of (a)-(d), case (ii) of condition $\left(\mathrm{D}_{3}\right)$ can be written $k=h-i-j$, where $h$ is the Coxeter number of $\mathfrak{g}$, and that in case (b) (resp. (c)), condition (ii)' can be written $k=\check{h}-i-j($ resp. $2 \breve{h}-i-j$ ), where $h$ is the dual Coxeter number of $\mathfrak{g}$. (This mysterious factor of 2 in the $C_{n}$ case is apparently well known to physicists.)

2. If $\mathfrak{g}$ is of type $D_{5}$, the triple $2,2,2$ satisfies $\left(\mathrm{CG}_{3}\right)$ (because $W\left(\lambda_{2}\right)$ is the adjoint $\mathfrak{g}$-module), but does not satisfy $\left(\mathrm{D}_{3}\right)$. Thus, the converse of 4.3 is false when $p=3$.

The proof of 4.6 is a straightforward, if tedious, computation. We discuss the example of $\mathfrak{g}=B_{4}$ to show what is involved. From [14], we see that $\tilde{\mathfrak{g}}$ is of type $A_{7}$ and $\sigma$ is the obvious involution: 
We take

$$
\gamma=s_{1} s_{2} s_{3} s_{4}, \quad \tilde{\gamma}=\tilde{s}_{1} \tilde{s}_{2} \tilde{s}_{3} \tilde{s}_{4} \sigma
$$

Then,

$$
\begin{gathered}
\phi_{1}=\alpha_{1}+\alpha_{2}+\alpha_{3}+2 \alpha_{4}, \quad \phi_{2}=\alpha_{2}+\alpha_{3}+2 \alpha_{4}, \quad \phi_{3}=\alpha_{3}+2 \alpha_{4}, \phi_{4}=\alpha_{4} \\
\tilde{\phi}_{1}=\tilde{\alpha}_{4}+\tilde{\alpha}_{5}+\tilde{\alpha}_{6}+\tilde{\alpha}_{7}, \tilde{\phi}_{2}=\tilde{\alpha}_{4}+\tilde{\alpha}_{5}+\tilde{\alpha}_{6}, \tilde{\phi}_{3}=\tilde{\alpha}_{4}+\tilde{\alpha}_{5}, \tilde{\phi}_{4}=\tilde{\alpha}_{4} .
\end{gathered}
$$

The orbits are as follows:

$$
\begin{aligned}
& R_{1}=\left\{ \pm \alpha_{1}, \pm \alpha_{2}, \pm \alpha_{3}, \pm\left(\alpha_{1}+\alpha_{2}+\alpha_{3}+2 \alpha_{4}\right)\right\} \\
& R_{2}=\left\{ \pm\left(\alpha_{1}+\alpha_{2}\right), \pm\left(\alpha_{2}+\alpha_{3}\right), \pm\left(\alpha_{2}+\alpha_{3}+2 \alpha_{4}\right)\right\} \\
& R_{3}=\left\{ \pm\left(\alpha_{3}+2 \alpha_{4}\right), \pm\left(\alpha_{1}+\alpha_{2}+\alpha_{3}\right), \pm\left(\alpha_{2}+2 \alpha_{3}+2 \alpha_{4}\right), \pm\left(\alpha_{1}+2 \alpha_{2}+2 \alpha_{3}+2 \alpha_{4}\right)\right\}, \\
& R_{4}=\left\{ \pm \alpha_{4}, \pm\left(\alpha_{3}+\alpha_{4}\right), \pm\left(\alpha_{2}+\alpha_{3}+\alpha_{4}\right), \pm\left(\alpha_{1}+\alpha_{2}+\alpha_{3}+\alpha_{4}\right)\right\} \\
& \tilde{R}_{1}=\left\{ \pm \alpha_{1}, \pm \alpha_{2}, \pm \alpha_{3}, \pm \alpha_{6}, \pm \alpha_{7}, \pm\left(\alpha_{4}+\alpha_{5}+\alpha_{6}+\alpha_{7}\right), \pm\left(\alpha_{1}+\alpha_{2}+\alpha_{3}+\alpha_{4}+\alpha_{5}\right)\right\}, \\
& \tilde{R}_{2}=\left\{ \pm\left(\alpha_{1}+\alpha_{2}\right), \pm\left(\alpha_{2}+\alpha_{3}\right), \pm\left(\alpha_{6}+\alpha_{7}\right), \pm\left(\alpha_{4}+\alpha_{5}+\alpha_{6}\right), \pm\left(\alpha_{2}+\alpha_{3}+\alpha_{4}+\alpha_{5}\right),\right. \\
&\left. \pm\left(\alpha_{3}+\alpha_{4}+\alpha_{5}+\alpha_{6}+\alpha_{7}\right), \pm\left(\alpha_{1}+\alpha_{2}+\alpha_{3}+\alpha_{4}+\alpha_{5}+\alpha_{6}\right)\right\}, \\
& \tilde{R}_{3}=\left\{ \pm\left(\alpha_{4}+\alpha_{5}\right), \pm\left(\alpha_{1}+\alpha_{2}+\alpha_{3}\right), \pm\left(\alpha_{3}+\alpha_{4}+\alpha_{5}\right), \pm\left(\alpha_{3}+\alpha_{4}+\alpha_{5}+\alpha_{6}\right),\right. \pm\left(\alpha_{2}+\alpha_{3}+\alpha_{4}+\alpha_{5}+\alpha_{6}\right), \pm\left(\alpha_{2}+\alpha_{3}+\alpha_{4}+\alpha_{5}+\alpha_{6}+\alpha_{7}\right), \\
&\left. \pm\left(\alpha_{1}+\alpha_{2}+\alpha_{3}+\alpha_{4}+\alpha_{5}+\alpha_{6}+\alpha_{7}\right)\right\}, \\
& \tilde{R}_{4}=\{ \pm \alpha_{4}, \pm \alpha_{5}, \pm\left(\alpha_{3}+\alpha_{4}\right), \pm\left(\alpha_{5}+\alpha_{6}\right), \pm\left(\alpha_{2}+\alpha_{3}+\alpha_{4}\right), \pm\left(\alpha_{5}+\alpha_{6}+\alpha_{7}\right), \\
&\left. \pm\left(\alpha_{1}+\alpha_{2}+\alpha_{3}+\alpha_{4}\right)\right\} .
\end{aligned}
$$

By inspection, one sees that $i, j, k$ satisfies $\left(\mathrm{D}_{3}\right)$ or $\left(\mathrm{TD}_{3}\right)$ exactly in the following cases:

$$
\left(\mathrm{D}_{3}\right):(1,1,2),(1,2,3),(1,4,4),(2,3,3),(2,4,4),(3,4,4) ;
$$

$\left(\mathrm{TD}_{3}\right):(1,1,2),(1,2,3),(1,3,3),(1,4,4),(2,2,3),(2,4,4),(3,4,4)$.

These results are in accordance with 4.6. 
In this section we collect some results which describe the restriction of $Y(\mathfrak{g})$-modules to 'diagram subalgebras' of $Y(\mathfrak{g})$.

Definition 5.1. Let $\emptyset \neq J \subseteq I$.

(i) $\mathfrak{g}_{J}$ is the Lie subalgebra of $\mathfrak{g}$ generated by the $H_{i}$ and the $X_{i}^{ \pm}$for $i \in J$;

(ii) $Y_{J}$ is the subalgebra of $Y(\mathfrak{g})$ generated by the $H_{i, k}$ and the $X_{i, k}^{ \pm}$, for $i \in J$, $k \in \mathbb{N}$.

(iii) $Q_{J}=\sum_{i \in J} \mathbb{Z} . \alpha_{i}, \quad Q_{J}^{+}=\sum_{i \in J} \mathbb{N} \cdot \alpha_{i}$.

It is clear from 2.2 that there is an algebra homomorphism $Y\left(\mathfrak{g}_{J}\right) \rightarrow Y_{J}$ which maps $H_{i, k} \mapsto H_{i, k}$ and $X_{i, k}^{ \pm} \mapsto X_{i, k}^{ \pm}$, for all $i \in J, k \in \mathbb{N}$. In particular, every $Y(\mathfrak{g})$ module may be regarded as a $Y\left(\mathfrak{g}_{J}\right)$-module. If $V$ is a highest weight $Y(\mathfrak{g})$-module with highest weight vector $v$, set

$$
V_{J}=Y\left(\mathfrak{g}_{J}\right) \cdot v .
$$

Note that $V_{J}$ is preserved by the action of $\mathfrak{h}$, since $\left[\mathfrak{h}, Y_{J}\right] \subseteq Y_{J}$.

Lemma 5.2. Let $\emptyset \neq J \subseteq I$.

(i) Let $V$ be a highest weight $Y(\mathfrak{g})$-module with highest weight $\lambda \in P^{+}$(as a $\mathfrak{g}$ module). Then,

$$
V_{J}=\bigoplus_{\eta \in Q_{J}^{+}} V_{\lambda-\eta} .
$$

(ii) If $V$ is an irreducible $Y(\mathfrak{g})$-module, then $V_{J}$ is an irreducible $Y\left(\mathfrak{g}_{J}\right)$-module. (iii) If $V$ and $W$ are irreducible $Y(\mathfrak{g})$-modules with highest weights $\lambda$ and $\mu$, then,

$$
V_{J} \otimes W_{J}=\bigoplus_{\eta \in Q_{J}^{+}}(V \otimes W)_{\lambda+\mu-\eta}
$$

The proof is straightforward (see Lemma 4.3 in [7] for part (ii)).

The canonical map $Y\left(\mathfrak{g}_{J}\right) \rightarrow Y(\mathfrak{g})$ is not a homomorphism of Hopf algebras. Nevertheless, we have

Lemma 5.3. Let $V$ and $W$ be finite-dimensional irreducible $Y(\mathfrak{g})$-modules and let $\emptyset \neq J \subseteq I$. Then, $V_{J} \otimes W_{J}$ is a $Y\left(\mathfrak{g}_{J}\right)$-submodule of $V \otimes W$.

This is Lemma 2.15 from [7]. The following is a more precise result.

Lemma 5.4. Let $U, V$ and $W$ be finite-dimensional irreducible $Y(\mathfrak{g})$-modules with highest weights (as $\mathfrak{g}$-modules) $\lambda$, $\mu$ and $\nu$, respectively, and let $\emptyset \neq J \subseteq I$.

(i) Assume that $\lambda+\mu-\nu \in Q_{J}^{+}$. Then, any non-zero $Y(\mathfrak{g})$-module homomorphism $U \otimes V \rightarrow W$ maps $U_{J} \otimes V_{J}$ onto $W_{J}$. In fact, this restriction defines an injective linear map

$$
\operatorname{Hom}_{Y(\mathfrak{g})}(U \otimes V, W) \rightarrow \operatorname{Hom}_{Y\left(\mathfrak{g}_{J}\right)}\left(U_{J} \otimes V_{J}, W_{J}\right) .
$$

(ii) Assume that $U_{J} \otimes V_{J}$ is a highest weight $Y\left(\mathfrak{g}_{J}\right)$-module and that $U \otimes V$ has an irreducible quotient $Y(\mathfrak{g})$-module with highest weight $\nu<\lambda+\mu$. Then, $\lambda+\mu-\nu \in$ $Q^{+} \backslash Q_{J}^{+}$.

Proof. The fact that any $Y(\mathfrak{g})$-module homomorphism $f: U \otimes V \rightarrow W$ maps $U_{J} \otimes V_{J}$ into $W_{J}$ follows from 5.2 (i) and (iii). If $f \neq 0$, the image of $f$ contains a $Y(\mathfrak{g})$ - 
restriction of $f$ to $U_{J} \otimes W_{J}$. By 5.2 (ii), $f$ is surjective, and the linear map (25) is injective.

Part (ii) follows immediately from part (i).

Lemma 5.5. Let $V$ and $W$ be finite-dimensional irreducible $Y(\mathfrak{g})$-modules, and let $\emptyset \neq J \subseteq I$. Assume that $V_{J} \otimes W_{J}$ contains a non-zero $Y\left(\mathfrak{g}_{J}\right)$-highest weight vector $u$ which is also an $\mathfrak{h}$-eigenvector of weight $\nu \in P^{+}$. Then, $(V \otimes W)_{\nu}$ contains a $Y(\mathfrak{g})$-highest weight vector.

Proof. Clearly, $\lambda+\mu-\nu \in Q_{J}^{+}$. It follows that $u \in(V \otimes W)_{\nu}^{++}$. The result now follows from the discussion preceding 3.1.

\section{The $A_{n}$ case}

In this section $\mathfrak{g}$ is of type $A_{n}(n \geq 1)$. The Coxeter number $h$ of $\mathfrak{g}$ is $n+1$. Proposition 4.6 implies that Conjecture 4.5 is a special case of

Theorem 6.1. Let $1 \leq i, j, k \leq n, a, b, c \in \mathbb{C}$. Then,

$$
\operatorname{Hom}_{Y(\mathfrak{g})}\left(V_{a}\left(\lambda_{i}\right) \otimes V_{b}\left(\lambda_{j}\right), V_{c}\left(\lambda_{k}\right)\right) \neq 0
$$

if and only if one of the following holds:

(i) $i+j<n+1, k=i+j, b-a=\frac{1}{2}(i+j), c-a=\frac{1}{2} j$;

(ii) $i+j>n+1, k=i+j-n-1, b-a=n+1-\frac{1}{2}(i+j), c-a=\frac{1}{2}(n+1-j)$.

Remark. It follows from 6.3 (i) below that the space

$$
\operatorname{Hom}_{Y(\mathfrak{g})}\left(V_{a}\left(\lambda_{i}\right) \otimes V_{b}\left(\lambda_{j}\right), V_{c}\left(\lambda_{k}\right)\right)
$$

is one-dimensional when it is non-zero.

We shall also prove the following:

Theorem 6.2. Let $1 \leq i \leq j \leq n, a, b \in \mathbb{C}$. Then, $V_{a}\left(\lambda_{i}\right) \otimes V_{b}\left(\lambda_{j}\right)$ is not a highest weight $Y(\mathfrak{g})$-module if and only if

$$
b-a=\frac{1}{2}(j-i)+r \quad \text { for some } 0<r \leq \min (i, n+1-j) .
$$

Hence, $V_{a}\left(\lambda_{i}\right) \otimes V_{b}\left(\lambda_{j}\right)$ is reducible as a $Y(\mathfrak{g})$-module if and only if

$$
b-a= \pm\left(\frac{1}{2}(j-i)+r\right) \quad \text { for some } 0<r \leq \min (i, n+1-j)
$$

Remark. One can show further that, when $b-a=\frac{1}{2}(j-i)+r$ for some $0<r \leq$ $\min (i, n+1-j)$, the $Y(\mathfrak{g})$-module $V_{a}\left(\lambda_{i}\right) \otimes V_{b}\left(\lambda_{j}\right)$ has a Jordan-Hölder series of length two: 
where $V$ is an irreducible $Y(\mathfrak{g})$-module such that

$$
V \cong \bigoplus_{s=0}^{r-1} W\left(\lambda_{i-s}+\lambda_{j+s}\right)
$$

as $\mathfrak{g}$-modules.

We begin with the following.

Proposition 6.3. Let $1 \leq i, j, k \leq n$.

(i) We have

$$
\operatorname{Hom}_{\mathfrak{g}}\left(W\left(\lambda_{i}\right) \otimes W\left(\lambda_{j}\right), W\left(\lambda_{k}\right)\right)=\left\{\begin{array}{l}
\mathbb{C} \text { if } k=i+j \text { or } k=i+j-n-1 \\
0 \text { otherwise. }
\end{array}\right.
$$

(ii) As $\mathfrak{g}$-modules, we have

$$
V_{a}\left(\lambda_{i}\right) \cong W\left(\lambda_{i}\right)
$$

(iii) Let $a, b, c \in \mathbb{C}$. Then,

$$
\operatorname{Hom}_{Y(\mathfrak{g})}\left(V_{a}\left(\lambda_{i}\right) \otimes V_{b}\left(\lambda_{j}\right), V_{c}\left(\lambda_{k}\right)\right)=0
$$

if $k \neq i+j$ or $i+j-n-1$. If $k=i+j$ or $i+j-n-1$, and $a$ and $b$ are fixed, the space

$$
\operatorname{Hom}_{Y(\mathfrak{g})}\left(V_{a}\left(\lambda_{i}\right) \otimes V_{b}\left(\lambda_{j}\right), V_{c}\left(\lambda_{k}\right)\right) \neq 0
$$

for at most one value of $c$, in which case it is one-dimensional.

Proof. Part (i) is easy, part (ii) is well known (see [11] and [8]), and part (iii) is immediate from parts (i) and (ii).

Proof of 6.1. By induction on $n$. The case $n=1$ is proved in [6]. Twisting by $\varphi$ and using 3.5 , we see that

$$
\operatorname{Hom}_{Y(\mathfrak{g})}\left(V_{a}\left(\lambda_{i}\right) \otimes V_{b}\left(\lambda_{j}\right), V_{c}\left(\lambda_{k}\right)\right) \neq 0
$$

if and only if

$$
\operatorname{Hom}_{Y(\mathfrak{g})}\left(V_{\frac{1}{2}(n+3)-b}\left(\lambda_{n+1-j}\right) \otimes V_{\frac{1}{2}(n+3)-a}\left(\lambda_{n+1-i}\right), V_{\frac{1}{2}(n+3)-c}\left(\lambda_{n+1-k}\right)\right) \neq 0 .
$$

Hence it suffices to prove the theorem when $i+j<n+1$.

Assume that

$$
\operatorname{Hom}_{Y(\mathfrak{g})}\left(V_{a}\left(\lambda_{i}\right) \otimes V_{b}\left(\lambda_{j}\right), V_{c}\left(\lambda_{k}\right)\right) \neq 0
$$

Since $i+j<n, 6.3$ implies that $k=i+j$. Noting that

$$
\lambda_{i}+\lambda_{j}-\lambda_{i+j} \in Q_{J}^{+}
$$

where $J=\{1,2, \ldots, i+j-1\}, 5.4$ gives 
whence $b-a=\frac{1}{2}(i+j)$ by 3.6 .

The value of $c$ can be computed as follows. Using 3.5 and 3.6, (27) implies that

$$
\operatorname{Hom}_{Y(\mathfrak{g})}\left(V_{a+\frac{1}{2}(i+j)}\left(\lambda_{j}\right), V_{a+\frac{1}{2}(n+1)}\left(\lambda_{n+1-i}\right) \otimes V_{c}\left(\lambda_{i+j}\right)\right) \neq 0
$$

and hence, by taking left duals, that

$$
\operatorname{Hom}_{Y(\mathfrak{g})}\left(V_{c-\frac{1}{2}(n+1)}\left(\lambda_{n+1-i-j}\right) \otimes V_{a}\left(\lambda_{i}\right), V_{a-\frac{1}{2}(n+1-i-j)}\left(\lambda_{n+1-j}\right)\right) \neq 0 .
$$

The first part of the proof now shows that

$$
a-\left(c-\frac{1}{2}(n+1)\right)=\frac{1}{2}(i+n+1-i-j)
$$

i.e.

$$
c-a=\frac{1}{2} j
$$

For the 'if' part, suppose that $k=i+j, b-a=\frac{1}{2}(i+j)$ and $c-a=\frac{1}{2} j$. Using 5.4 with $J=\{n-i-j-2, \ldots, n\}$ and 5.5 , we see that

$$
\left(V_{b-\frac{1}{2}(n+1)}\left(\lambda_{n+1-j}\right) \otimes V_{a-\frac{1}{2}(n+1)}\left(\lambda_{n+1-i}\right)\right)_{\lambda_{n+1-i-j}^{+}}^{+} \neq 0
$$

Since there is no non-zero dominant weight strictly less than $\lambda_{n+1-i-j}$, it follows that for some $c^{\prime} \in \mathbb{C}$,

$$
\operatorname{Hom}_{Y(\mathfrak{g})}\left(V_{c^{\prime}-\frac{1}{2}(n+1)}\left(\lambda_{n+1-i-j}\right), V_{b-\frac{1}{2}(n+1)}\left(\lambda_{n+1-j}\right) \otimes V_{a-\frac{1}{2}(n+1)}\left(\lambda_{n+1}\right)\right) \neq 0 .
$$

Applying 3.5 and 3.6 shows that

$$
\operatorname{Hom}_{Y(\mathfrak{g})}\left(V_{a}\left(\lambda_{i}\right) \otimes V_{b}\left(\lambda_{j}\right), V_{c^{\prime}}\left(\lambda_{k}\right)\right) \neq 0
$$

But then, by 6.3 (iii), $c^{\prime}$ is uniquely determined, and by the 'only if' part, $c^{\prime}=c$.

The proof of 6.1 is now complete.

Proof of 6.2. By induction on $n$. If $n=1$, the result is contained in [6]. Assuming the result is known when $\mathfrak{g}$ is of type $A_{m}$ for $m<n$, we prove it when $\mathfrak{g}$ is of type $A_{n}$ by induction on $\min (i, n+1-j)$. If $i=1$ or $j=n$, the result follows from 6.1 and 6.3 , since

$$
\begin{aligned}
& W\left(\lambda_{1}\right) \otimes W\left(\lambda_{j}\right) \cong W\left(\lambda_{1}+\lambda_{j}\right) \oplus W\left(\lambda_{j+1}\right), \\
& W\left(\lambda_{n}\right) \otimes W\left(\lambda_{j}\right) \cong W\left(\lambda_{n}+\lambda_{j}\right) \oplus W\left(\lambda_{j-1}\right) .
\end{aligned}
$$

Assume now that $\min (i, n+1-j)>1$. To prove the 'only if' part of 6.2 , consider the case $i+j<n+1$ (resp. the case $i+j>n+1$ ).

Since $V_{a}\left(\lambda_{i}\right) \otimes V_{b}\left(\lambda_{j}\right)$ is not a highest weight $Y(\mathfrak{g})$-module, there exists an irreducible $Y(\mathfrak{g})$-module $V$ with $\mathfrak{g}$-highest weight $\lambda=\lambda_{i}+\lambda_{j}-\eta$, for some $0 \neq \eta \in Q^{+}$, such that

$$
\operatorname{Hom}_{Y(\mathfrak{g})}\left(V_{a}\left(\lambda_{i}\right) \otimes V_{b}\left(\lambda_{j}\right), V\right) \neq 0 .
$$

By 3.5, 3.6 and 6.1, we have 
(resp.

$$
\left.\operatorname{Hom}_{Y(\mathfrak{g})}\left(V_{a}\left(\lambda_{i}\right) \otimes V_{b-\frac{1}{2}}\left(\lambda_{j+1}\right), V \otimes V_{b-\frac{1}{2}(j+1)}\left(\lambda_{1}\right)\right) \neq 0\right) .
$$

If $b-a \neq \frac{1}{2}(j-i)+r$ for any $1<r \leq i$, then by the induction hypothesis on $\min (i, n+1-j), V_{a+\frac{1}{2}}\left(\lambda_{i-1}\right) \otimes V_{b}\left(\lambda_{j}\right)$ (resp. $\left.V_{a}\left(\lambda_{i}\right) \otimes V_{b-\frac{1}{2}}\left(\lambda_{j+1}\right)\right)$ is a highest weight $Y(\mathfrak{g})$-module, so

$$
\lambda_{i-1}+\lambda_{j} \leq \lambda_{n}+\lambda_{i}+\lambda_{j}-\eta
$$

i.e.

$$
\eta \leq \alpha_{i}+\cdots+\alpha_{n}
$$

(resp.

$$
\lambda_{i}+\lambda_{j+1} \leq \lambda_{1}+\lambda_{i}+\lambda_{j}-\eta
$$

i.e.

$$
\left.\eta \leq \alpha_{1}+\cdots+\alpha_{j}\right)
$$

This, together with the requirement that $\lambda \in P^{+}$, forces $\eta=\alpha_{i}+\cdots+\alpha_{j}$, so $\lambda=\lambda_{i-1}+\lambda_{j+1}$. Noting that $\lambda_{i}+\lambda_{j}-\lambda_{i-1}-\lambda_{j+1} \in Q_{J}^{+}$, where $J=\{i, i+1, \ldots, j\}$, it follows from 5.4 that

$$
\operatorname{Hom}_{Y\left(\mathfrak{g}_{J}\right)}\left(V_{a}\left(\lambda_{1}\right) \otimes V_{b}\left(\lambda_{j-i+1}\right), \mathbb{C}\right) \neq 0 .
$$

By 3.6, we see that $b-a=\frac{1}{2}(j-i)+1$, as required.

We now prove the 'if' part of 6.2 , assuming it when $\mathfrak{g}$ is of type $A_{m}$ for $m<n$, and for smaller values of $\min (i, n-j+1)$ when $\mathfrak{g}$ is of type $A_{n}$. We consider three cases.

Suppose first that $i+j<n+1$ (resp. $i+j>n+1)$. Let

$$
b-a=\frac{1}{2}(j-i)+r \text { for some } 0<r \leq i,
$$

and assume for a contradiction that $V_{a}\left(\lambda_{i}\right) \otimes V_{b}\left(\lambda_{j}\right)$ is a highest weight $Y(\mathfrak{g})$-module. Let $J^{\prime}=\{1,2, \ldots, n-1\}$ (resp. $\left.J^{\prime}=\{2, \ldots, n\}\right)$. Since $V_{a}\left(\lambda_{i}\right) \otimes V_{b}\left(\lambda_{j}\right)$ is assumed to be $Y(\mathfrak{g})$-highest weight,

$$
V_{a}\left(\lambda_{i}\right)_{J^{\prime}} \otimes V_{b}\left(\lambda_{j}\right)_{J^{\prime}} \subset Y(\mathfrak{g}) \cdot\left(v_{i} \otimes v_{j}\right),
$$

where $v_{i}$ and $v_{j}$ are $Y(\mathfrak{g})$-highest weight vectors in $V_{a}\left(\lambda_{i}\right)$ and $V_{b}\left(\lambda_{j}\right)$. But then 5.2 implies that

$$
V_{a}\left(\lambda_{i}\right)_{J^{\prime}} \otimes V_{b}\left(\lambda_{j}\right)_{J^{\prime}} \subset Y\left(\mathfrak{g}_{J^{\prime}}\right) \cdot\left(v_{i} \otimes v_{j}\right),
$$

and hence that $V_{a}\left(\lambda_{i}\right)_{J^{\prime}} \otimes V_{b}\left(\lambda_{j}\right)_{J^{\prime}}$ is $Y\left(\mathfrak{g}_{J^{\prime}}\right)$-highest weight. By the induction hypothesis on $n, b-a$ cannot take any of the values in (28). This is the desired contradiction.

If $i+j=n+1$, the argument used above fails when $b-a=\frac{1}{2}(n+1)$, since in that case $V_{a}\left(\lambda_{i}\right)_{J^{\prime}} \otimes V_{b}\left(\lambda_{j}\right)_{J^{\prime}}$ is $Y\left(\mathfrak{g}_{J^{\prime}}\right)$-highest weight. But for this value of $b-a$, the contradiction is immediate from 3.6.

We have now completely proved Theorem 6.2, except for the final statement, which follows immediately from 3.6 and 3.8 .

\section{The $D_{n}$ case}

In this section $\mathfrak{g}$ is of type $D_{n},(n \geq 4)$. The Coxeter number $h$ is $2 n-2$. Conjecture 
Theorem 7.1. Let $1 \leq i \leq j \leq k \leq n, a, b, c \in \mathbb{C}$. Then,

$$
\operatorname{Hom}_{Y(\mathfrak{g})}\left(V_{a}\left(\lambda_{i}\right) \otimes V_{b}\left(\lambda_{j}\right), V_{c}\left(\lambda_{k}\right)\right) \neq 0
$$

if and only if one of the following holds:

(i) $i+j \leq n-2, k=i+j, b-a=\frac{1}{2}(i+j), c-a=\frac{1}{2} j$;

(ii) $i+j \geq n, j \leq n-2, k=2 n-i-j-2, b-a=\frac{1}{2}(i+j), c-a=\frac{1}{2} j$;

(iii) $i \leq n-2, j=n-1, b-a=\frac{1}{2}(n+i-1), c-a=\frac{1}{2}(n-i-1)$,

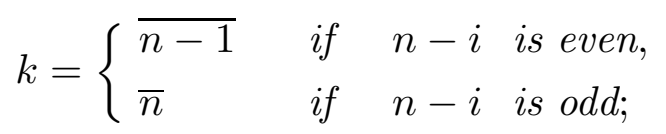

(iv) $i \leq n-2, j=n, b-a=\frac{1}{2}(n+i-1), c-a=\frac{1}{2}(n-i-1)$,

$$
k= \begin{cases}\bar{n} & \text { if } n-i \text { is even } \\ \overline{n-1} & \text { if } n-i \text { is odd }\end{cases}
$$

Moreover, the space of homomorphisms in (26) is one-dimensional when it is nonzero.

We shall also prove

Theorem 7.2. Let $1 \leq i \leq j \leq n$. Then, $V_{a}\left(\lambda_{i}\right) \otimes V_{b}\left(\lambda_{j}\right)$ is not a highest weight $Y(\mathfrak{g})$-module if and only if one of the following holds:

(i) $j \leq n-2$,

$$
b-a= \begin{cases}\frac{1}{2}(j-i)+r & \text { for some } 0<r \leq \min (i, n-j), \text { or } \\ n-1-r-\frac{1}{2}(j-i) & \text { for some } 0 \leq r<\min (i, n-j)\end{cases}
$$

(ii) $i \leq n-2, j=n-1$ or $n$,

$$
b-a=\frac{1}{2}(n-1-i)+r \text { for some } 0<r \leq i
$$

(iii) $i=j=n-1$ or $n$,

$$
b-a=n-r-1 \quad \text { for some } 0 \leq r \leq n-2 \text { with } n-r \text { even; }
$$

(iv) $i=n-1, j=n$,

$$
b-a=n-r-1 \quad \text { for some } 0 \leq r \leq n-2 \text { with } n-r \text { odd. }
$$

Hence, $V_{a}\left(\lambda_{i}\right) \otimes V_{b}\left(\lambda_{j}\right)$ is reducible as a $Y(\mathfrak{g})$-module if and only if $\pm(b-a)$ takes one of the above values.

We first recall from [7], Theorem 6.2 , the $\mathfrak{g}$-module structure of the fundamental 
Proposition 7.3. Let $a \in \mathbb{C}$. Then as a $\mathfrak{g}$-module,

$$
V_{a}\left(\lambda_{i}\right) \cong \begin{cases}\bigoplus_{k=0}^{\left[\frac{i}{2}\right]} W\left(\lambda_{i-2 k}\right) & \text { if } i \leq n-2, \\ W\left(\lambda_{i}\right) & \text { if } i=n-1 \text { or } n\end{cases}
$$

where $\lambda_{0}=0$.

We first prove

Proposition 7.4. Let $1 \leq j \leq n-2$ and let $v_{1}$ and $v_{j}$ be $Y(\mathfrak{g})$-highest weight vectors in $V\left(\lambda_{1}\right)$ and $V\left(\lambda_{j}\right)$, respectively.

(i) $V_{a}\left(\lambda_{1}\right) \otimes V_{b}\left(\lambda_{j}\right)$ is not a $Y(\mathfrak{g})$-highest weight module if and only if $b-a=\frac{1}{2}(j+1)$ or $n-\frac{1}{2}(j+1)$.

(ii) If $b-a=\frac{1}{2}(j+1)$ or $\left.n-\frac{1}{2}(j+1)\right)$, then $V_{a}\left(\lambda_{1}\right) \otimes V_{b}\left(\lambda_{j}\right)$ has a Jordan-Hölder series of length two, namely

$$
0 \rightarrow Y(\mathfrak{g}) \cdot\left(v_{1} \otimes v_{j}\right) \rightarrow V_{a}\left(\lambda_{1}\right) \otimes V_{b}\left(\lambda_{j}\right) \rightarrow V_{a+\frac{1}{2} j}\left(\lambda_{j+1}\right) \rightarrow 0
$$

or

$$
\left.0 \rightarrow Y(\mathfrak{g}) \cdot\left(v_{1} \otimes v_{j}\right) \rightarrow V_{a}\left(\lambda_{1}\right) \otimes V_{b}\left(\lambda_{j}\right) \rightarrow V_{a+n-1-\frac{1}{2} j}\left(\lambda_{j-1}\right) \rightarrow 0\right),
$$

respectively (if $j=n-2$, the first short exact sequence should be replaced by

$$
\left.0 \rightarrow Y(\mathfrak{g}) \cdot\left(v_{1} \otimes v_{j}\right) \rightarrow V_{a}\left(\lambda_{1}\right) \otimes V_{b}\left(\lambda_{j}\right) \rightarrow V_{a+\frac{1}{2}(n-2)}\left(\lambda_{n-1}\right) \otimes V_{a+\frac{1}{2}(n-2)}\left(\lambda_{n}\right) \rightarrow 0\right)
$$

Proof. Let $b-a=\frac{1}{2}(j+1)$ and $J=\{1,2, \ldots, n-2\}$. By 6.1, the $Y(\mathfrak{g})$-submodule $V_{b}\left(\lambda_{j}\right)_{J} \otimes V_{a}\left(\lambda_{1}\right)_{J}$ of $V_{b}\left(\lambda_{j}\right) \otimes V_{a}\left(\lambda_{1}\right)$ has a $Y\left(\mathfrak{g}_{J}\right)$-highest weight vector of weight $\lambda_{j+1}=\lambda_{1}+\lambda_{j}-\alpha_{1}-\cdots-\alpha_{j}$ for $\mathfrak{g}$. Hence, by $5.5, V_{b}\left(\lambda_{j}\right) \otimes V_{a}\left(\lambda_{1}\right)$ has a $Y(\mathfrak{g})-$ highest weight vector of weight $\lambda_{j+1}$. But then, by 3.14, $V_{a}\left(\lambda_{1}\right) \otimes V_{b}\left(\lambda_{j}\right)$ cannot be $Y(\mathfrak{g})$-highest weight.

For the converse, assume that $V_{a}\left(\lambda_{1}\right) \otimes V_{b}\left(\lambda_{j}\right)$ is not $Y(\mathfrak{g})$-highest weight, let $M=V_{a}\left(\lambda_{1}\right) \otimes V_{b}\left(\lambda_{j}\right) / Y(\mathfrak{g}) \cdot\left(v_{1} \otimes v_{j}\right)$, let $N$ be an irreducible quotient of $M$, and let $\lambda \in P^{+}$be the maximal weight of $N$ as a $\mathfrak{g}$-module. The dominant weights $\lambda<\lambda_{1}+\lambda_{j}$ are of two types:

(i) $j \geq 3, \lambda=\lambda_{1}+\lambda_{j-2 k}, 0<k \leq[j / 2]$, and

(ii) $\lambda=\lambda_{j+1-2 k}, 0 \leq k \leq[(j+1) / 2]$,

with the understanding that $\lambda_{0}=0$. In case (i),

$$
\operatorname{Hom}_{Y(\mathfrak{g})}\left(V_{a}\left(\lambda_{1}\right) \otimes V_{b}\left(\lambda_{j}\right), N\right) \neq 0
$$

which implies that

$$
\operatorname{Hom}_{Y(\mathfrak{g})}\left(V_{b}\left(\lambda_{j}\right), V_{a+n-1}\left(\lambda_{1}\right) \otimes N\right) \neq 0
$$

and hence that $\lambda_{j} \leq 2 \lambda_{1}+\lambda_{j-2 k}$. This implies that $k=1$, i.e. $\lambda=\lambda_{1}+\lambda_{j-2}$. But then $\lambda_{1}+\lambda_{j}-\lambda \in Q_{J^{\prime}}^{+}$, where $J^{\prime}=\{j-1, j, \ldots, n\}$. Hence, 5.5 implies that 
which is absurd. Thus, case (ii) must hold. As above, one sees that $k=0$ or 1 (and $k=1$ if $j=n-2$ ), so we must have either

(iia) $\operatorname{Hom}_{Y(\mathfrak{g})}\left(V_{a}\left(\lambda_{1}\right) \otimes V_{b}\left(\lambda_{j}\right), V_{c}\left(\lambda_{j+1}\right)\right) \neq 0$ (if $j<n-2$ ), or

(iib) $\operatorname{Hom}_{Y(\mathfrak{g})}\left(V_{a}\left(\lambda_{1}\right) \otimes V_{b}\left(\lambda_{j}\right), V_{c}\left(\lambda_{j-1}\right)\right) \neq 0$,

for some $c \in \mathbb{C}$.

In case (iia), note that $\lambda_{1}+\lambda_{j}-\lambda_{j+1}=\alpha_{1}+\cdots+\alpha_{j} \in Q_{J^{\prime \prime}}^{+}$, where $J^{\prime \prime}=$ $\{1,2, \ldots, n-2\}$. Hence,

$$
\operatorname{Hom}_{Y\left(\mathfrak{g}_{J^{\prime \prime}}\right)}\left(V_{a}\left(\lambda_{1}\right)_{J^{\prime \prime}} \otimes V_{b}\left(\lambda_{j}\right)_{J^{\prime \prime}}, V_{c}\left(\lambda_{j+1}\right)_{J^{\prime \prime}}\right) \neq 0
$$

which gives $b-a=\frac{1}{2}(j+1)$ and $c-a=\frac{1}{2} j$, by 6.1 .

In case (iib), we get

$$
\operatorname{Hom}_{Y(\mathfrak{g})}\left(V_{b}\left(\lambda_{j}\right), V_{a+n-1}\left(\lambda_{1}\right) \otimes V_{c}\left(\lambda_{j-1}\right)\right) \neq 0
$$

and hence, taking left duals,

$$
\operatorname{Hom}_{Y(\mathfrak{g})}\left(V_{c-n+1}\left(\lambda_{j-1}\right) \otimes V_{a}\left(\lambda_{1}\right), V_{b-n+1}\left(\lambda_{j}\right)\right) \neq 0
$$

Finally, twisting with $\varphi$ gives

$$
\operatorname{Hom}_{Y(\mathfrak{g})}\left(V_{n-a}\left(\lambda_{1}\right) \otimes V_{2 n-1-c}\left(\lambda_{j-1}\right), V_{2 n-1-b}\left(\lambda_{j}\right)\right) \neq 0 .
$$

We are now in the situation of (iia). Hence,

$$
2 n-1-c-(n-a)=\frac{1}{2} j \text { and } 2 n-1-b-(n-a)=\frac{1}{2}(j-1),
$$

i.e.

$$
b-a=n-\frac{1}{2}(j+1) \text { and } \quad c-a=n-1-\frac{1}{2} j .
$$

We have now proved (i). In fact, the preceding argument shows that, if $V$ is an irreducible quotient of $V_{a}\left(\lambda_{1}\right) \otimes V_{b}\left(\lambda_{j}\right)$ with highest weight different from $\lambda_{1}+\lambda_{j}$, then either

$$
\begin{gathered}
b-a=\frac{1}{2}(i+j) \text { and } V \cong V_{a+\frac{1}{2} j}\left(\lambda_{j+1}\right), \text { or } \\
b-a=n-\frac{1}{2}(j+1) \text { and } V \cong V_{a+n-1-\frac{1}{2} j}\left(\lambda_{j-1}\right) .
\end{gathered}
$$

We prove part (ii) of 7.4 when $b-a=\frac{1}{2}(j+1)$; the other case is similar. First, if $N$ is any $Y(\mathfrak{g})$-submodule (or quotient module) of $V_{a}\left(\lambda_{1}\right) \otimes V_{b}\left(\lambda_{j}\right)$, then, since $\operatorname{Hom}_{Y(\mathfrak{g})}\left(V_{a-n+1}\left(\lambda_{1}\right) \otimes N, V_{b}\left(\lambda_{j}\right)\right) \neq 0,\left(\operatorname{resp} . \operatorname{Hom}_{Y(\mathfrak{g})}\left({ }^{t} N \otimes V_{a}\left(\lambda_{1}\right), V_{b-n+1}\left(\lambda_{j}\right)\right) \neq\right.$ $0)$, we see by using 7.3 that

$$
m_{0}(N) \neq 0 \text { if } j \text { is odd, and } m_{1}(N) \neq 0 \text { if } j \text { is even. }
$$

If $L=Y(\mathfrak{g}) \cdot\left(v_{1} \otimes v_{j}\right)$ is reducible for $Y(\mathfrak{g})$, let $L^{\prime} \subset L$ be an irreducible $Y(\mathfrak{g})$ submodule. Then, $L^{\prime} \cong V_{e}\left(\lambda_{j-1}\right)$ for some $e \in \mathbb{C}$, and we get 
But this is impossible when $b-a=\frac{1}{2}(j+1)$, by $(29)$.

Let $M$ be the quotient $V_{a}\left(\lambda_{1}\right) \otimes V_{b}\left(\lambda_{j}\right) / L$. Then, $M=Y(\mathfrak{g}) \cdot M_{\lambda_{j+1}}$, since otherwise $M$ would have an irreducible quotient which would have to be of highest weight $\lambda_{j-1}$, and we have seen above that this is impossible for this value of $b-a$. Since $M$ is non-zero, this shows that $M_{\lambda_{j+1}} \neq 0$. On the other hand, since $M_{\lambda_{j+1}} \subseteq M^{+}$, we have

$$
\operatorname{dim}\left(M_{\lambda_{j+1}}\right) \leq m_{\lambda_{j+1}}\left(V_{a}\left(\lambda_{1}\right) \otimes V_{b}\left(\lambda_{j}\right)\right) .
$$

This multiplicity is one, and so $M_{\lambda_{j+1}}$ is one-dimensional. Thus, $M$ is a highest weight $Y(\mathfrak{g})$-module with $\mathfrak{g}$-highest weight $\lambda_{j+1}$. If $M$ is not irreducible for $Y(\mathfrak{g})$, it contains an irreducible $Y(\mathfrak{g})$-submodule, which must be of the form $V_{d}\left(\lambda_{j+1-2 k}\right)$ for some $1 \leq k \leq[(j+1) / 2], d \in \mathbb{C}$. By 7.3, this means that $m_{\lambda_{1}}(M)=2$ if $j$ is even, and $m_{0}(M)=2$ if $j$ is odd. But this would mean that $m_{0}(L)=0$ or $m_{\lambda_{1}}(L)=0$, and we have seen that this is impossible.

To prove 7.1 , we need

Proposition 7.5. Let $1 \leq i \leq n-2, a \in \mathbb{C}$.

(i) If $n-i$ is even,

$\operatorname{Hom}_{Y(\mathfrak{g})}\left(V_{a}\left(\lambda_{n}\right) \otimes V_{b}\left(\lambda_{n}\right), V_{c}\left(\lambda_{i}\right)\right) \neq 0 \quad$ iff $b-a=n-i-1, c-a=\frac{1}{2}(n-i-1)$,
$\operatorname{Hom}_{Y(\mathfrak{g})}\left(V_{a}\left(\lambda_{n-1}\right) \otimes V_{b}\left(\lambda_{n-1}\right), V_{c}\left(\lambda_{i}\right)\right) \neq 0 \quad$ iff $b-a=n-i-1, c-a=\frac{1}{2}(n-i-1)$.

(ii) If $n-i$ is odd,

$\operatorname{Hom}_{Y(\mathfrak{g})}\left(V_{a}\left(\lambda_{n-1}\right) \otimes V_{b}\left(\lambda_{n}\right), V_{c}\left(\lambda_{i}\right)\right) \neq 0 \quad$ iff $\quad b-a=n-i-1, \quad c-a=\frac{1}{2}(n-i-1)$, $\operatorname{Hom}_{Y(\mathfrak{g})}\left(V_{a}\left(\lambda_{n}\right) \otimes V_{b}\left(\lambda_{n-1}\right), V_{c}\left(\lambda_{i}\right)\right) \neq 0 \quad$ iff $b-a=n-i-1, \quad c-a=\frac{1}{2}(n-i-1)$.

Proof. We prove the first statement in part (i); the proofs in the other cases are similar. In [7], Proposition 6.2, we established that, if $b-a=n-i-1$, there exist $c, c^{\prime} \in \mathbb{C}$ such that

$\operatorname{Hom}_{Y(\mathfrak{g})}\left(V_{a}\left(\lambda_{n}\right) \otimes V_{b}\left(\lambda_{n}\right), V_{c}\left(\lambda_{i}\right)\right) \neq 0$ and $\operatorname{Hom}_{Y(\mathfrak{g})}\left(V_{a}\left(\lambda_{n-1}\right) \otimes V_{b}\left(\lambda_{n-1}\right), V_{c^{\prime}}\left(\lambda_{i}\right)\right) \neq 0$.

To see that $c=c^{\prime}=a+\frac{1}{2}(n-i-1)$, notice that since $m_{\lambda_{i}}\left(V\left(\lambda_{n}\right) \otimes V\left(\lambda_{n}\right)\right)=1$, the values of $c$ and $c^{\prime}$ are uniquely determined by $a$ and $b$. But now, twisting by $\varphi$ and applying $\tau_{a+b}$ gives

$$
\operatorname{Hom}_{Y(\mathfrak{g})}\left(V_{a}\left(\lambda_{n}\right) \otimes V_{b}\left(\lambda_{n}\right), V_{a+b-c}\left(\lambda_{i}\right)\right) \neq 0
$$

and

$$
\operatorname{Hom}_{Y(\mathfrak{g})}\left(V_{a}\left(\lambda_{n-1}\right) \otimes V_{b}\left(\lambda_{n-1}\right), V_{a+b-c^{\prime}}\left(\lambda_{i}\right)\right) \neq 0
$$

and hence

$$
a+b-c=c \text { and } a+b-c^{\prime}=c^{\prime} .
$$

Conversely, suppose that $\operatorname{Hom}_{Y(\mathfrak{g})}\left(V_{a}\left(\lambda_{n}\right) \otimes V_{b}\left(\lambda_{n}\right), V_{c}\left(\lambda_{i}\right)\right) \neq 0$. We prove by 
follows from 7.4 (i) by using a diagram automorphism of order three of $Y(\mathfrak{g})$, so the induction begins. Assume the result when $\mathfrak{g}$ is of type $D_{m}$ with $m<n$. Now, $2 \lambda_{n}-\lambda_{i} \in Q_{J}^{+}$, where $J=\{2,3, \ldots, n\}$, so by the induction hypothesis on $n$, we get

$$
b-a=n-1-(i-1)-1=n-i-1 .
$$

The value of $c-a$ is determined as before.

Proof of Theorem 7.1. We only have to prove the theorem in cases (i) and (ii), since 7.5 establishes cases (iii) and (iv).

The 'only if' part is proved by by induction on $n$. The induction actually begins at $n=3$, when $\mathfrak{g}$ is of type $A_{3}$, and the result in that case is contained in 6.1 . Assume now that $n \geq 4$ and that the result is known when $\mathfrak{g}$ is of type $D_{m}$ for $m<n$.

Suppose then that

$$
\operatorname{Hom}_{Y(\mathfrak{g})}\left(V_{a}\left(\lambda_{i}\right) \otimes V_{b}\left(\lambda_{j}\right), V_{c}\left(\lambda_{k}\right)\right) \neq 0
$$

This implies by Proposition 7.4 that

$$
\operatorname{Hom}_{Y(\mathfrak{g})}\left(V_{a-\frac{1}{2}}\left(\lambda_{i-1}\right) \otimes V_{a+\frac{1}{2}(i-1)}\left(\lambda_{1}\right) \otimes V_{b}\left(\lambda_{j}\right), V_{c}\left(\lambda_{k}\right)\right) \neq 0
$$

and hence

$$
\operatorname{Hom}_{Y(\mathfrak{g})}\left(V_{a+\frac{1}{2}(i-1)}\left(\lambda_{1}\right) \otimes V_{b}\left(\lambda_{j}\right), V_{a+n-\frac{3}{2}}\left(\lambda_{i-1}\right) \otimes V_{c}\left(\lambda_{k}\right)\right) \neq 0
$$

Let $F$ be a non-zero element in $\operatorname{Hom}_{Y(\mathfrak{g})}\left(V_{a+\frac{1}{2}(i-1)}\left(\lambda_{1}\right) \otimes V_{b}\left(\lambda_{j}\right), V_{a+n-\frac{3}{2}}\left(\lambda_{i-1}\right) \otimes V_{c}\left(\lambda_{k}\right)\right)$, and let $v_{1}$ and $v_{j}$ be $Y(\mathfrak{g})$-highest weight vectors in $V_{a+\frac{1}{2}(i-1)}\left(\lambda_{1}\right)$ and $V_{b}\left(\lambda_{j}\right)$, respectively. We first prove that one of the following must hold:

$(\alpha) b-a=\frac{1}{2}(i+j)$ and $F\left(v_{1} \otimes v_{j}\right)=0$;

( $\beta) b-a=n-1-\frac{1}{2}(j-i)$ and $F\left(v_{1} \otimes v_{j}\right)=0$;

$(\gamma) F\left(v_{1} \otimes v_{j}\right) \neq 0, i+j \leq n, k=i+j-2, b-a=\frac{1}{2}(i+j)-1, c-a=\frac{1}{2} j$

( $\delta) F\left(v_{1} \otimes v_{j}\right) \neq 0, i+j \geq n+2, k=2 n-i-j, b-a=\frac{1}{2}(i+j)-1, c-a=\frac{1}{2} j$.

If $F\left(v_{1} \otimes v_{j}\right)=0$, then, by 7.4 , we see that either $(\alpha)$ or $(\beta)$ must hold. On the other hand, if $F\left(v_{1} \otimes v_{j}\right) \neq 0$, then, using the fact that $\lambda_{i-1}+\lambda_{k}-\lambda_{1}-\lambda_{j} \in Q_{J}^{+}$, where $J=\{2,3, \ldots, n\}$, we see from 5.5 that, if $i>2$,

$$
\operatorname{Hom}_{Y\left(\mathfrak{g}_{J}\right)}\left(V_{a+\frac{1}{2}}\left(\lambda_{i-2}\right) \otimes V_{b}\left(\lambda_{j-1}\right), V_{c}\left(\lambda_{k-1}\right)\right) \neq 0
$$

The induction hypothesis on $n$ now shows that either $(\gamma)$ or $(\delta)$ must hold. If $i=1$, the same conclusion follows from 7.4. Finally, if $i=2$, we get $b=c$ and $j=k$, so

$$
F: V_{a+\frac{1}{2}}\left(\lambda_{1}\right) \otimes V_{b}\left(\lambda_{j}\right) \rightarrow V_{a+n-\frac{3}{2}}\left(\lambda_{1}\right) \otimes V_{b}\left(\lambda_{j}\right)
$$

Since $a+\frac{1}{2} \neq a+n-\frac{3}{2}, 3.2$ implies that $F\left(v_{1} \otimes v_{j}\right)=0$, contradicting our assumption. 
Next, we prove that (30) implies that $(\alpha)$ must hold. Observe that if we twist by $\varphi$ and apply $\tau_{a+b-n}$, then (30) implies that

$$
\operatorname{Hom}_{Y(\mathfrak{g})}\left(V_{a}\left(\lambda_{j}\right) \otimes V_{b}\left(\lambda_{i}\right), V_{a+b-c}\left(\lambda_{k}\right)\right) \neq 0
$$

Suppose that $a, b$ and $c$ satisfy the conditions in $(\gamma)$ or $(\delta)$ above. Then, it is easy to see that $a, b$ and $a+b-c$ do not satisfy any of the conditions $(\alpha)-(\delta)$. Thus, the only possibilities are $(\alpha)$ and $(\beta)$. We prove by induction on $i$ that $(\beta)$ is impossible.

If $i=1$, we know by 7.4 that $k=j+1$ ( since $i \leq j \leq k)$ and that $b-a=\frac{1}{2}(j+1)$, so $(\beta)$ is impossible in this case. Assume that $(\alpha)$ is the only possibility for for $i-1$. If $(\beta)$ holds for $i$, we see from 7.4 that

$$
\operatorname{Hom}_{Y(\mathfrak{g})}\left(V_{a+n-1-\frac{1}{2}(j-i+1)}\left(\lambda_{j-1}\right), V_{a+n-\frac{3}{2}}\left(\lambda_{i-1}\right) \otimes V_{c}\left(\lambda_{k}\right)\right) \neq 0,
$$

or equivalently that

$$
\operatorname{Hom}_{Y(\mathfrak{g})}\left(V_{a-\frac{1}{2}}\left(\lambda_{i-1}\right) \otimes V_{a+n-1-\frac{1}{2}(j-i+1)}\left(\lambda_{j-1}\right), V_{c}\left(\lambda_{k}\right)\right) \neq 0 .
$$

Since $(\alpha)$ holds for $i-1$, we get

$$
a+n-1-\frac{1}{2}(j-i+1)-\left(a-\frac{1}{2}\right)=\frac{1}{2}(i+j-2),
$$

i.e. $j=n$, contradicting our assumption that $j<n$. This completes the induction, and proves that (30) implies $(\alpha)$.

We now show, again by induction on $i$, that (30) implies that either (i) or (ii) in the statement of 7.1 must hold. If $i=1$, the result follows from 7.4.

Assume the result for $i-1$. To complete the induction we consider four cases:

Case 1. $j<k \leq n-2$. By 7.4, $(\alpha)$ gives $k=i+j$ or $2 n-i-j-2$,

$$
\operatorname{Hom}_{Y(\mathfrak{g})}\left(V_{a-\frac{1}{2}}\left(\lambda_{i-1}\right) \otimes V_{a+\frac{1}{2}(i+j-1)}\left(\lambda_{j+1}\right), V_{c}\left(\lambda_{k}\right)\right) \neq 0
$$

and

$$
c-\left(a-\frac{1}{2}\right)=\frac{1}{2}(j+1)
$$

i.e. $c-a=\frac{1}{2} j$. Thus, either (i) or (ii) in 7.1 must hold for $i$.

Case 2. $j=k<n-2$. Again, $(\alpha)$ gives

$$
\operatorname{Hom}_{Y(\mathfrak{g})}\left(V_{a-\frac{1}{2}}\left(\lambda_{i-1}\right) \otimes V_{a+\frac{1}{2}(i+j-1)}\left(\lambda_{j+1}\right), V_{c}\left(\lambda_{j}\right)\right) \neq 0
$$

Unfortunately, 7.1 does not apply to this because $j+1 \not \leq j$. However, the nonvanishing of this last space of homomorphisms is equivalent to

$$
\operatorname{Hom}_{Y(\mathfrak{g})}\left(V_{c-n+1}\left(\lambda_{j}\right) \otimes V_{a-\frac{1}{2}}\left(\lambda_{i-1}\right), V_{a+\frac{1}{2}(i+j-1)-n+1}\left(\lambda_{j+1}\right)\right) \neq 0
$$

and hence, twisting by $\varphi$ and then applying $\tau_{n-1}$, to 
We can apply the induction hypothesis to this inequality, and this gives that $j+1=$ $i-1+j$ or $2 n-(i-1)-j-2$, and

$-c+n-1-\left(-a+\frac{1}{2}\right)=\frac{1}{2}(i-1+j)$ and $-a-\frac{1}{2}(i+j-1)+n-1-\left(-a+\frac{1}{2}\right)=\frac{1}{2} j$.

In both cases, we get $i+2 j=2 n-2$ and $c-a=\frac{1}{2} j$, so 7.1 (ii) is satisfied (we already know that $\left.b-a=\frac{1}{2}(i+j)\right)$.

Case 3. $i<n-2, j=k=n-2$. We show that this case is possible only if $i=2$ (it is obvious that $i$ must be even). We first determine the value of $c$. Observe that (30) implies that

$$
\operatorname{Hom}_{Y(\mathfrak{g})}\left(V_{c-n+1}\left(\lambda_{n-2}\right) \otimes V_{a}\left(\lambda_{i}\right), V_{b-n+1}\left(\lambda_{n-2}\right)\right) \neq 0,
$$

and hence, twisting by $\varphi$ and applying $\tau_{n-1}$, we get

$$
\operatorname{Hom}_{Y(\mathfrak{g})}\left(V_{-a}\left(\lambda_{i}\right) \otimes V_{-c+n-1}\left(\lambda_{n-2}\right), V_{-b+n-1}\left(\lambda_{n-2}\right)\right) \neq 0
$$

Since $(\alpha)$ must hold for this, we get

$$
c=a+\frac{1}{2}(n-i)
$$

By 7.4, we see that (30) implies

$\operatorname{Hom}_{Y(\mathfrak{g})}\left(V_{a-n+\frac{1}{2}(i+3)}\left(\lambda_{1}\right) \otimes V_{a+\frac{1}{2}}\left(\lambda_{i+1}\right) \otimes V_{a+\frac{1}{2}(n+i-2)}\left(\lambda_{n-2}\right), V_{a+\frac{1}{2}(n-i)}\left(\lambda_{n-2}\right)\right) \neq 0$, which gives

$$
\operatorname{Hom}_{Y(\mathfrak{g})}\left(V_{a+\frac{1}{2}}\left(\lambda_{i+1}\right) \otimes V_{a+\frac{1}{2}(n+i-2)}\left(\lambda_{n-2}\right), V_{a+\frac{1}{2}(i+1)}\left(\lambda_{1}\right) \otimes V_{a+\frac{1}{2}(n-i)}\left(\lambda_{n-2}\right)\right) \neq 0
$$

By 7.4 again, the module on the right-hand side of this space of homomorphisms is irreducible. Thus, if $v_{1}$ and $v_{n-2}$ are $Y(\mathfrak{g})$-highest weight vectors in $V_{a+\frac{1}{2}(i+1)}\left(\lambda_{1}\right)$ and $V_{a+\frac{1}{2}(n-i)}\left(\lambda_{n-2}\right)$, respectively, $v_{1} \otimes v_{n-2}$ must be in the image of any non-zero homomorphism $F$ in

$$
\operatorname{Hom}_{Y(\mathfrak{g})}\left(V_{a+\frac{1}{2}}\left(\lambda_{i+1}\right) \otimes V_{a+\frac{1}{2}(n+i-2)}\left(\lambda_{n-2}\right), V_{a+\frac{1}{2}(i+1)}\left(\lambda_{1}\right) \otimes V_{a+\frac{1}{2}(n-i)}\left(\lambda_{n-2}\right)\right) .
$$

Since $\lambda_{i+1}-\lambda_{1} \in Q_{J}^{+}$, where $J=\{2,3, \ldots, n\}$, we see from 5.5 that

$$
\operatorname{Hom}_{Y\left(\mathfrak{g}_{J}\right)}\left(V_{a+\frac{1}{2}}\left(\lambda_{i}\right) \otimes V_{a+\frac{1}{2}(n+i-2)}\left(\lambda_{n-3}\right), V_{a+\frac{1}{2}(n-i)}\left(\lambda_{n-3}\right)\right) \neq 0 .
$$

The induction hypothesis on $n$ now proves that $i=2$.

Case 4. $i=j=k=n-2$. In this case, we have $b=a+n-2$ and $c=a+\frac{1}{2}(n-2)$. Equation (30) implies that

$$
\operatorname{Hom}_{Y(\mathfrak{g})}\left(V_{c-n+1}\left(\lambda_{n-2}\right) \otimes V_{a}\left(\lambda_{n-2}\right), V_{b-n+1}\left(\lambda_{n-2}\right)\right) \neq 0 .
$$


Finally, the inductive step, and with it the proof of the 'only if' part of 7.1 , is complete.

We now prove the 'if' part. Suppose that (i) holds. Taking $J=\{1,2, \ldots, n-2\}$, we see that $V_{a+\frac{1}{2}(i+j)}\left(\lambda_{j}\right) \otimes V_{\frac{1}{2} a}\left(\lambda_{i}\right)$ has a $Y(\mathfrak{g})$-highest weight vector of weight $\lambda_{i+j}$. This vector cannot generate an irreducible highest weight $Y(\mathfrak{g})$-submodule, since otherwise it would have an irreducible $Y(\mathfrak{g})$-submodule, which would necessarily be of the form $V_{c}\left(\lambda_{r}\right)$ for some $r<i+j$, and this is impossible by the 'only if' part of 7.1 .

Suppose now that (ii) holds. Recall from the discussion in Section 4 that we may assume that $i \leq j \leq k$.

Consider first the case when $n, i$ and $j$ are all even. By 7.5,

$$
\begin{aligned}
& \operatorname{Hom}_{Y(\mathfrak{g})}\left(V_{a-\frac{1}{2}(n+i-1)}\left(\lambda_{n}\right) \otimes V_{a}\left(\lambda_{i}\right), V_{a-\frac{1}{2}(n-i-1)}\left(\lambda_{n}\right)\right) \neq 0, \\
& \operatorname{Hom}_{Y(\mathfrak{g})}\left(V_{a-\frac{1}{2}(n-i-1)}\left(\lambda_{n}\right), V_{a+j-\frac{1}{2}(n+i-1)}\left(\lambda_{n}\right) \otimes V_{a+\frac{1}{2}(i+j)-n+1}\left(\lambda_{j}\right)\right) \neq 0 .
\end{aligned}
$$

Hence,

$$
\operatorname{Hom}_{Y(\mathfrak{g})}\left(V_{a-\frac{1}{2}(n+i-1)}\left(\lambda_{n}\right) \otimes V_{a}\left(\lambda_{i}\right), V_{a+j-\frac{1}{2}(n-i-1)}\left(\lambda_{n}\right) \otimes V_{a+\frac{1}{2}(i+j)-n+1}\left(\lambda_{j}\right)\right) \neq 0
$$

or equivalently,

$$
\operatorname{Hom}_{Y(\mathfrak{g})}\left(V_{a}\left(\lambda_{i}\right) \otimes V_{a+\frac{1}{2}(i+j)}\left(\lambda_{j}\right), V_{a+\frac{1}{2}(n-i-1)}\left(\lambda_{n}\right) \otimes V_{a+j-\frac{1}{2}(n-i-1)}\left(\lambda_{n}\right)\right) \neq 0 .
$$

We consider the composite of a non-zero element $F$ of this space homomorphisms with the non-zero homomorphism

$$
V_{a+\frac{1}{2}(n-i-1)}\left(\lambda_{n}\right) \otimes V_{a+j-\frac{1}{2}(n-i-1)}\left(\lambda_{n}\right) \rightarrow V_{a+\frac{1}{2} j}\left(\lambda_{2 n-2-i-j}\right)
$$

given by 7.5. This composite cannot be zero, otherwise the image of $F$ would be a $Y(\mathfrak{g})$-module $N$ for which

$$
\operatorname{Hom}_{Y(\mathfrak{g})}\left({ }^{t} N \otimes V_{a}\left(\lambda_{i}\right), V_{a+\frac{1}{2}(i+j)-n+1}\left(\lambda_{j}\right)\right) \neq 0
$$

Moreover, the irreducible $\mathfrak{g}$-modules occurring in $N$ would be among the set

$$
\left\{W\left(2 \lambda_{n}\right), W\left(\lambda_{n-2}\right), W\left(\lambda_{n-4}\right), \ldots, W\left(\lambda_{2 n-i-j}\right)\right\}
$$

Since $i \leq k=2 n-i-j-2$,

$$
m_{0}\left({ }^{t} N \otimes W\left(\lambda_{i}\right)\right)=0, \quad m_{0}\left(W\left(\lambda_{j}\right)\right)=1,
$$

so (31) is impossible.

The proofs in the other cases are similar applications of 7.5 and the $\mathfrak{g}$-module decomposition of the fundamental $Y(\mathfrak{g})$-modules. We omit the details.

This completes the proof of the 'if' part of 7.1 .

It remains to give the

Proof of 7.2. We proceed by induction on $n$. As usual, the induction starts at $n=3$, where the result is known from 6.2. Assume now that 7.2 is known when $\mathfrak{g}$ 
first the case when $i \leq j \leq n-2$, and proceed by induction on $\min (i, n-j)$. The induction starts when $i=1$, this case being covered by 7.4.

Assume that $V_{a}\left(\lambda_{i}\right) \otimes V_{b}\left(\lambda_{j}\right)$ is not $Y(\mathfrak{g})$-highest weight. Let $v_{i}$ and $v_{j}$ be $Y(\mathfrak{g})$ highest weight vectors in $V\left(\lambda_{i}\right)$ and $V\left(\lambda_{j}\right)$, respectively, and let $N$ be an irreducible $Y(\mathfrak{g})$-quotient of $V\left(\lambda_{i}\right) \otimes V\left(\lambda_{j}\right) / Y(\mathfrak{g}) \cdot\left(v_{i} \otimes v_{j}\right)$. Then, we have

$$
\operatorname{Hom}_{Y(\mathfrak{g})}\left(V_{a}\left(\lambda_{i}\right) \otimes V_{b}\left(\lambda_{j}\right), N\right) \neq 0
$$

and $N_{\lambda_{i}+\lambda_{j}}=0$. Assume for a contradiction that $b-a$ takes none of the values

or

$$
\frac{1}{2}(j-i)+r, \quad 0<r \leq \min (i, n-j)
$$

$$
n-1+\frac{1}{2}(i-j)-r, \quad 0 \leq r<\min (i, n-j) .
$$

If $i+j \leq n$, we use 7.4 to get

$$
\operatorname{Hom}_{Y(\mathfrak{g})}\left(V_{a+\frac{1}{2}(1-i)}\left(\lambda_{1}\right) \otimes V_{a+\frac{1}{2}}\left(\lambda_{i-1}\right) \otimes V_{b}\left(\lambda_{j}\right), N\right) \neq 0
$$

and hence

$$
\operatorname{Hom}_{Y(\mathfrak{g})}\left(V_{a+\frac{1}{2}}\left(\lambda_{i-1}\right) \otimes V_{b}\left(\lambda_{j}\right), V_{a+n-\frac{1}{2}(i+1)}\left(\lambda_{1}\right) \otimes N\right) \neq 0
$$

By the assumption on $b-a$ and the induction hypothesis on $\min (i, n-j), V_{a}\left(\lambda_{i-1}\right) \otimes V_{b}\left(\lambda_{j}\right)$ is $Y(\mathfrak{g})$-highest weight. Hence, $\lambda_{1}+\lambda \geq \lambda_{i-1}+\lambda_{j}$, so $\lambda=\lambda_{i}+\lambda_{j}-\eta$, where $\eta=\sum_{i=1}^{n} r_{i} \alpha_{i} \in Q^{+}$satisfies

$$
\eta \leq \alpha_{1}+2 \alpha_{2}+\cdots+\alpha_{i-1}+2\left(\alpha_{i}+\cdots+\alpha_{n-2}\right)+\alpha_{n-1}+\alpha_{n}
$$

If $r_{1}=0$, let $J=\{2,3, \ldots, n\}$. Then, by 5.4 ,

$$
\operatorname{Hom}_{Y\left(\mathfrak{g}_{J}\right)}\left(V_{a}\left(\lambda_{i-1}\right) \otimes V_{b}\left(\lambda_{j-1}\right), N_{J}\right) \neq 0 .
$$

By the induction hypothesis on $n$, we have

$$
b-a=\left\{\begin{array}{l}
\frac{1}{2}(j-i)+r \text { for some } 0<r \leq i-1, \text { or } \\
n-2+\frac{1}{2}(i-j)-r \text { for some } 0 \leq r<i-1 .
\end{array}\right.
$$

But all these values have beeen excluded.

Using $\lambda \in P^{+}$, one sees that the case $r_{1}>0$ is possible only if $i=2$, and then either $\eta=\lambda_{2}$, or $\eta \in Q_{J^{\prime}}^{+}$, where $J^{\prime}=\{1,2, \ldots, n-2, n-1\}$ or $\{1,2, \ldots, n-2, n\}$. In the first case, $\lambda=\lambda_{j}$ and (32) becomes

$$
\operatorname{Hom}_{Y(\mathfrak{g})}\left(V_{a+\frac{1}{2}}\left(\lambda_{1}\right) \otimes V_{b}\left(\lambda_{j}\right), V_{a+n-\frac{1}{2}(i+1)}\left(\lambda_{1}\right) \otimes V_{c}\left(\lambda_{j}\right)\right) \neq 0
$$

for some $c \in \mathbb{C}$. By the assumption on $b-a, V_{a+\frac{1}{2}}\left(\lambda_{1}\right) \otimes V_{b}\left(\lambda_{j}\right)$ is $Y(\mathfrak{g})$-highest weight, but then (33) contradicts 3.2 . In the second case, 5.4 gives 
and then 6.2 gives

$$
b-a=\frac{1}{2} j \text { or } \frac{1}{2} j+1 .
$$

Both of these values have been excluded.

Thus, we have obtained the desired contradiction when $i+j \leq n$. If $i+j>n$, one uses a similar argument, but using 7.4 to replace (32) by

$$
\operatorname{Hom}_{Y(\mathfrak{g})}\left(V_{a}\left(\lambda_{i}\right) \otimes V_{b-\frac{1}{2}}\left(\lambda_{j+1}\right), N \otimes V_{b+\frac{1}{2}(1-j)}\left(\lambda_{1}\right)\right) \neq 0
$$

This proves the 'only if' part of 7.2 when $j \leq n-2$.

For the 'if' part, note that the $i=1$ case is contained in 7.4. Suppose that $i>1$. If $b-a=\frac{1}{2}(j-i)+r$, where $0<r \leq \min (i, n-j)$, let $J^{\prime \prime}=\{1,2, \ldots, n-1\}$. By 6.2 , $V_{a}\left(\lambda_{i}\right)_{J^{\prime \prime}} \otimes V_{b}\left(\lambda_{j}\right)_{J^{\prime \prime}}$ is not $Y(\mathfrak{g})$-highest weight, so by 5.5 , neither is $V_{a}\left(\lambda_{i}\right) \otimes V_{b}\left(\lambda_{j}\right)$. If $b-a=n-i+\frac{1}{2}(i-j)-r$, where $0<r<\min (i, n-j)$, one uses the same argument with $J^{\prime \prime}$ replaced by $\{2,3, \ldots, n\}$ and uses the induction hypothesis on $n$ instead of $6.2 \ldots$ For the remaining value $b-a=n-1+\frac{1}{2}(i-j)$, note that, if $i \neq j$, we have

$$
\operatorname{Hom}_{Y(\mathfrak{g})}\left(V_{a}\left(\lambda_{i}\right) \otimes V_{a+n-1+\frac{1}{2}(i-j)}\left(\lambda_{j}\right), V_{a+n-1-\frac{1}{2}(j)}\left(\lambda_{j-i}\right)\right) \neq 0
$$

by 7.1 , while if $i=j$, then by 3.4 and 3.6 , we have

$$
\operatorname{Hom}_{Y(\mathfrak{g})}\left(V_{a}\left(\lambda_{i}\right) \otimes V_{a+n-1}\left(\lambda_{i}\right), \mathbb{C}\right) \neq 0
$$

In any case, this implies that $V_{a}\left(\lambda_{i}\right) \otimes V_{b}\left(\lambda_{j}\right)$ is not $Y(\mathfrak{g})$-highest weight.

We now consider part (ii). If $j=n$, the result follows by the above argument, using 5.5 and 6.2 (and the same $J^{\prime \prime}$ ). If $j=n-1$, replace $J^{\prime \prime}$ with $\{1,2, \ldots, n-2, n\}$.

Finally, parts (iii) and (iv) follow immediately from 7.5 .

\section{The $B_{n}$ and $C_{n}$ cases}

In this section, we give the analogues of Theorems 6.1 and 7.1 when $\mathfrak{g}$ is of type $B_{n}$ or $C_{n}$.

Theorem 8.1. Let $\mathfrak{g}$ be of type $B_{n}$, and let $1 \leq i \leq j \leq k \leq n, a, b, c \in \mathbb{C}$. Then,

$$
\operatorname{Hom}_{Y(\mathfrak{g})}\left(V_{a}\left(\lambda_{i}\right) \otimes V_{b}\left(\lambda_{j}\right), V_{c}\left(\lambda_{k}\right)\right) \neq 0
$$

if and only if one of the following holds:

(i) $i+j \leq n-1, k=i+j, b-a=i+j, c-a=j$;

(ii) $i<n, j=k=n, b-a=n+i-1, c-a=n-i-1$.

The proof of this theorem is very similar to that of 7.1. Although we shall omit the details, we remark that the argument used to prove the existence of a non-zero homomorphism of $Y(\mathfrak{g})$-modules 
(for suitable $a, b, c$ ) when $\mathfrak{g}$ is of type $D_{n}$ fails to produce a non-zero homomorphism

$$
V_{a}\left(\lambda_{i}\right) \otimes V_{b}\left(\lambda_{j}\right) \rightarrow V_{c}\left(\lambda_{2 n-i-j}\right)
$$

when $\mathfrak{g}$ is of type $B_{n}$ (as would be predicted by condition (D) alone) because of a difference in the way that tensor products of spin modules for $\mathfrak{g}$ behave in the two cases. Namely, in the $B_{n}$ case, the tensor product of the spin module with itself contains every fundamental $\mathfrak{g}$-module except the spin module, whereas in the $D_{n}$ case, the tensor product of the two spin modules, or of a spin module with itself, contains only 'half' the remaining fundamental $\mathfrak{g}$-modules.

Theorem 8.2. Let $\mathfrak{g}$ be of type $C_{n}$, and let $1 \leq i \leq j \leq k \leq n, a, b, c \in \mathbb{C}$. Then,

$$
\operatorname{Hom}_{Y(\mathfrak{g})}\left(V_{a}\left(\lambda_{i}\right) \otimes V_{b}\left(\lambda_{j}\right) \otimes V_{c}\left(\lambda_{k}\right), \mathbb{C}\right) \neq 0
$$

if and only if $i+j \leq n, k=i+j, b-a=\frac{1}{2}(i+j), c-a=\frac{1}{2} j$.

The proof of this theorem is similar to that of 6.1. Note that the fundamental $Y(\mathfrak{g})$-modules are irreducible as $\mathfrak{g}$-modules in both the $A_{n}$ and $C_{n}$ cases (see [11] and $[8])$.

Remark. We can use $6.1,7.1,8.1$ and 8.2 to prove 4.2 , avoiding the use of the PRV conjecture. In fact, we can prove a more general result. Suppose, for example, that $\mathfrak{g}$ is of type $D_{n}$ and that $i, j \leq n-2, a, b \in \mathbb{C}$. Let $V$ be any irreducible quotient $Y(\mathfrak{g})$-module of $V_{a}\left(\lambda_{i}\right) \otimes V_{b}\left(\lambda_{j}\right)$, and let $\lambda$ be the highest weight of $V$ as a $\mathfrak{g}$-module. Then,

$$
\operatorname{Hom}_{\mathfrak{g}}\left(W\left(\lambda_{i}\right) \otimes W\left(\lambda_{j}\right), W(\lambda)\right) \neq 0 .
$$

Indeed, since $\lambda$ is dominant and $\leq \lambda_{i}+\lambda_{j}$, we have either

(i) $\lambda=\lambda_{k}$ for some $k$, or

(ii) $\lambda=\lambda_{k}+\lambda_{\ell}$ for some $k, \ell$.

In case (i), we know that $(i, j, k)$ satisfies the conditions in 4.5 , and then (34) is easily checked. In case (ii), $\lambda_{i}+\lambda_{j}-\lambda_{k}-\lambda_{\ell} \in Q_{J}^{+}$, where $J=\{2,3, \ldots, n\}$, so by 5.4 and an obvious induction on $n$, we have

$$
\operatorname{Hom}_{\mathfrak{g}_{J}}\left(W\left(\lambda_{i}\right)_{J} \otimes W\left(\lambda_{j}\right)_{J}, W(\lambda)_{J}\right) \neq 0,
$$

which implies

$$
\operatorname{Hom}_{\mathfrak{g}}\left(W\left(\lambda_{i}\right) \otimes W\left(\lambda_{j}\right), W(\lambda)\right) \neq 0 .
$$

Similar arguments apply in the other cases.

\section{The quantum affine case}

In this section, we indicate how to translate the preceding results from the context of 
in [8], Chapter 12. We assume throughout that the deformation parameter $\epsilon$ is not a root of unity.

To find the quantum affine version of 6.1 , for example, one replaces $Y(\mathfrak{g})$ by $U_{\epsilon}(\hat{\mathfrak{g}}), V_{a}\left(\lambda_{i}\right)$ by $V_{\epsilon}\left(\lambda_{i}, a\right)$, etc., and conditions such as

$$
b-a=\frac{1}{2}(i+j), \quad c-a=\frac{1}{2} j
$$

in 6.1 (i) by

$$
b / a=\epsilon^{i+j}, \quad c / a=\epsilon^{j} .
$$

Similarly, in 6.2 , the condition for $V_{\epsilon}\left(\lambda_{i}, a\right) \otimes V_{\epsilon}\left(\lambda_{j}, b\right)$ not to be a highest weight $U_{\epsilon}(\hat{\mathfrak{g}})$-module is

$$
b / a=\epsilon^{j-i+2 r} \quad \text { for some } 0<r \leq \min (i, n+1-j) .
$$

The main results in Sections 7 and 8 can be translated in the same way. We leave this to the reader, as well as the straightforward problem of appropriately reformulating the proofs.

\section{Appendix: Dorey's rule and affine Toda theories}

In this section, we sketch how Dorey's condition arises in the context of ATFTs. We shall consider only those ATFTs based on untwisted affine algebras.

We begin by summarizing some results related to Coxeter elements, for which we follow [15]. Let $\alpha_{0}=-\theta, X_{0}^{ \pm}=X_{\theta}^{\mp}$, and $\hat{I}=I \amalg\{0\}$. Let $k_{i}(i \in I)$ be the coprime positive integers such that

$$
\sum_{i \in \hat{I}} k_{i} \alpha_{i}=0
$$

(so that $k_{0}=1$ ), and set

$$
X^{ \pm}=\sum_{i \in \hat{I}} \sqrt{k_{i}} X_{i}^{ \pm} .
$$

Since $X^{+}$is a regular element [15], its centralizer is a Cartan subalgebra $\mathfrak{h}^{\prime}$ of $\mathfrak{g}$. Note that $\left[X^{+}, X^{-}\right]=0$, so $X^{ \pm} \in \mathfrak{h}^{\prime}$. Recall also that $h=\sum_{i \in \hat{I}} k_{i}$.

Let $H \in \mathfrak{h}$ be such that $\alpha_{i}(H)=1$ for all $i \in I$, and set

$$
A=\exp \left(\frac{2 \pi \sqrt{-1}}{h} H\right) .
$$

Thus, $A$ lies in a connected complex simple Lie group $G$ with Lie algebra $\mathfrak{g}$.

Note that the centralizer of $A$ in $\mathfrak{g}$ is $\mathfrak{h}$. On the other hand, it is clear that

$$
\operatorname{Ad}(A)\left(X^{ \pm}\right)=\omega^{ \pm 1} X^{ \pm}
$$

where $\omega=e^{2 \pi \sqrt{-1} / h}$. It follows that $\operatorname{Ad}(A)\left(\mathfrak{h}^{\prime}\right)=\mathfrak{h}^{\prime}$. In fact, it is known [15] that $\left.\operatorname{Ad}(A)\right|_{\mathfrak{h}^{\prime}}$ is a Coxeter transformation of $\mathfrak{h}^{\prime}$, i.e. one can choose an ordered set of simple roots $\alpha_{1}^{\prime}, \alpha_{2}^{\prime}, \ldots, \alpha_{n}^{\prime}$ of $\mathfrak{g}$ with respect to $\mathfrak{h}^{\prime}$ such that 
where $s_{i}^{\prime}$ is the $i$ th simple reflection in the Weyl group of $\mathfrak{g}$ with respect to $\mathfrak{h}^{\prime}$. Define, for $i \in I$,

$$
\phi_{i}^{\prime}=s_{n}^{\prime} s_{n-1}^{\prime} \ldots s_{i+1}^{\prime}\left(\alpha_{i}^{\prime}\right),
$$

and let $R_{i}^{\prime}$ be the $\gamma^{\prime}$-orbit of $\phi_{i}^{\prime}$.

Choose root vectors $X_{\alpha^{\prime}}$, for every root $\alpha^{\prime}$ of $\mathfrak{g}$ with respect to $\mathfrak{h}^{\prime}$, such that

$$
\operatorname{Ad}(A)\left(X_{\alpha^{\prime}}\right)=X_{\gamma^{\prime}\left(\alpha^{\prime}\right)}
$$

and set

$$
\tilde{H}_{i}=\sum_{\beta^{\prime} \in R_{i}^{\prime}} X_{\beta^{\prime}}
$$

It is clear that $\operatorname{Ad}(A)\left(\tilde{H}_{i}\right)=\tilde{H}_{i}$, so $\tilde{H}_{i} \in \mathfrak{h}$. Obviously, the $\tilde{H}_{i}$ are linearly independent, and hence form a basis of $\mathfrak{h}$.

We now turn to Toda field theory. The ATFT based on the affine Lie algebra $\hat{\mathfrak{g}}$ is defined by the lagrangian

$$
\mathcal{L}(\Psi)=\iint\left\{\left\|\frac{\partial \Psi}{\partial t}\right\|^{2}-\left\|\frac{\partial \Psi}{\partial x}\right\|^{2}+\frac{m^{2}}{\beta^{2}}\left(e^{\operatorname{ad}(\Psi)}\left(X^{+}\right), X^{-}\right)\right\} d x d t
$$

where $\Psi$ is a function of the coordinates $(x, t)$ on $1+1$ dimensional spacetime with values in $\mathfrak{h},($,$) is the invariant bilinear form on \mathfrak{g}, \mathrm{m}^{2}$ is a (positive) mass scale, and $\beta$ is a coupling constant (usually either real or purely imaginary). Since the $\tilde{H}_{i}$ are a basis of $\mathfrak{h}$, we can write

$$
\Psi=\sum_{i \in I} \psi_{i} \tilde{H}_{i}
$$

where the $\psi_{i}$ are scalar-valued functions. The component $\psi_{i}$ is associated with the $i$ th particle of the theory. The potential term

$$
V(\Psi)=\left(e^{\operatorname{ad}(\Psi)}\left(X^{+}\right), X^{-}\right)
$$

in $\mathcal{L}(\Psi)$ can be expanded formally as a power series in the $\psi_{i}$

$$
V(\Psi)=\sum_{p=0}^{\infty} \sum_{i_{1}, i_{2}, \ldots, i_{p} \in I} V_{i_{1} i_{2} \ldots i_{p}} \psi_{i_{1}} \psi_{i_{2}} \ldots \psi_{i_{p}}
$$

and one says that there is a coupling (or fusing) between the particles labelled $i_{1}, i_{2}, \ldots, i_{p}$ if $V_{i_{1} i_{2} \ldots i_{p}} \neq 0$.

From (36),

$$
V_{i_{1} i_{2} \ldots i_{p}}=\frac{1}{n !} \sum\left(\left[\tilde{H}_{i_{\pi(1)}},\left[\tilde{H}_{i_{\pi(2)}}, \ldots,\left[\tilde{H}_{i_{\pi(p)}}, X^{+}\right] \cdots\right]\right], X^{-}\right)
$$


where the sum is over all permutations $\pi$ of $\{1,2, \ldots, p\}$. Next, using (35), we get $(37)$

$$
V_{i_{1} i_{2} \ldots i_{r}}=\frac{1}{p !} \sum_{\pi} \sum_{\beta_{1}^{\prime} \in R_{i_{\pi(1)}^{\prime}}} \sum_{\beta_{2}^{\prime} \in R_{i_{\pi(2)}^{\prime}}^{\prime}} \ldots \sum_{\beta_{p}^{\prime} \in R_{i_{\pi(p)}^{\prime}}^{\prime}}\left(\left[X_{\beta_{1}^{\prime}},\left[X_{\beta_{2}^{\prime}}, \ldots,\left[X_{\beta_{p}^{\prime}}, X^{+}\right] \cdots\right]\right], X^{-}\right) .
$$

Since the weight of

$$
\left[X_{\beta_{1}^{\prime}},\left[X_{\beta_{2}^{\prime}}, \ldots,\left[X_{\beta_{p}^{\prime}}, X^{+}\right] \cdots\right]\right]
$$

with respect to $\mathfrak{h}^{\prime}$ is $\beta_{1}^{\prime}+\beta_{2}^{\prime}+\cdots+\beta_{p}^{\prime}$, it is clear that the term on the right-hand side of (37) corresponding to $\beta_{1}^{\prime}, \beta_{2}^{\prime}, \cdots, \beta_{p}^{\prime}$ can be non-zero only if

$$
\beta_{1}^{\prime}+\beta_{2}^{\prime}+\cdots+\beta_{p}^{\prime}=0
$$

and hence that

$$
V_{i_{1} i_{2} \ldots i_{p}} \neq 0 \text { only if } 0 \in R_{i_{1}}^{\prime}+R_{i_{2}}^{\prime}+\cdots+R_{i_{p}}^{\prime}
$$

Thus, the $p$-point coupling $V_{i_{1} i_{2} \ldots i_{p}} \neq 0$ only if $i_{1}, i_{2}, \ldots, i_{p}$ satisfies $\left(\mathrm{D}_{p}\right)$. The converse statement also holds when $p=3$, but this requires a case-by-case analysis, which we omit.

\section{References}

1. D. Bernard, Hidden Yangians in 2D massive current algebras, Commun. Math. Phys. 137 (1991), 191-208.

2. D. Bernard and A. LeClair, Quantum group symmetries and non-local currents in 2D QFT, Commun. Math. Phys. 142 (1991), 99-138.

3. N. Bourbaki, Eléments de Mathématique, Fasc. XXXIV, Groupes et Algebres de Lie, Ch. IV-VI, Hermann, Paris, 1968.

4. H. W. Braden, A note on affine Toda couplings, J. Phys. A 25 (1992), L15-L20.

5. H. W. Braden, E. Corrigan, P. E. Dorey and R. Sasaki, Affine Toda field theory and exact S-matrices, Nucl. Phys. B338 (1990), 689-746.

6. V. Chari and A. N. Pressley, Yangians and R-matrices, L'Enseign. Math. 36 (1990), 267-302.

7. V. Chari and A. N. Pressley, Fundamental representations of Yangians and singularities of R-matrices, J. reine angew. Math. 417 (1991), 87-128.

8. V. Chari and A. N. Pressley, A Guide to Quantum Groups, Cambridge University Press, Cambridge, 1994.

9. G. W. Delius, M. T. Grisaru and D. Zanon, Exact S-matrices for non-simplylaced affine Toda theories, Nucl. Phys. B382 (1992), 365-406.

10. P. E. Dorey, Root systems and purely elastic S-matrices, Nucl. Phys. B358 (1991), 654-676.

11. V. Drinfel'd, Hopf algebras and the quantum Yang-Baxter equation, Soviet Math. Dokl. 32 (1985), 254-258.

12. V. Drinfel'd, A new realization of Yangians and quantum affine algebras, Soviet 
13. L. D. Faddeev, Quantum completely integrable models in field theory, Soviet Scientific Reviews Sect. C 1, 107-155, Harwood Academic Publishers, Chur, Switzerland.

14. V. G. Kac, Infinite dimensional Lie algebras, Birkhäuser, Boston, 1983.

15. B. Kostant, The principal three-dimensional subgroup and the Betti numbers of a complex simple Lie group, Am. J. Math. 81 (1959), 973-1032.

16. S. Kumar, A proof of the Parthasarathy-Ranga Rao-Varadarajan conjecture, Invent. Math. 93 (1988), 117-130.

17. N. J. MacKay, The full set of $c_{n}$-invariant factorized S-matrices, J. Phys. A 25 (1992), L1343-L1349.

18. O. Mathieu, Construction d'un groupe de Kac-Moody et applications, Compositio Math. 69 (1989), 37-60.

19. E. Ogievetsky, N. Yu. Reshetikhin and P. Wiegmann, The principal chiral field in two dimensions on classical Lie algebras, Nucl. Phys. B280 (1987), 45-96.

20. D. I. Olive, N. Turok and J. W. R. Underwood, Affine Toda solitons and vertex operators, Nucl. Phys. B409 (1993), 509-546.

21. K. R. Parthasarathy, R. Ranga Rao and V. S. Varadarajan, Representations of complex semi-simple Lie groups and Lie algebras, Ann. Math. 85 (2) (1967), $383-429$.

22. T. A. Springer, Regular elements of finite reflection groups, Invent. Math. 25 (1974), 159-198.

23. R. Steinberg, Finite reflection groups, Trans. Am. Math. Soc. 91 (1959), 493-504.

\author{
Vyjayanthi Chari \\ Department of Mathematics \\ University of California \\ Riverside \\ CA 92521 \\ USA \\ email: chari@ucrmath.ucr.edu
}

\author{
Andrew Pressley \\ Department of Mathematics \\ King's College \\ Strand \\ London WC2R 2LS \\ UK \\ email:anp@uk.ac.kcl.mth
}

